TRANSACTIONS OF THE

AMERICAN MATHEMATICAL SOCIETY

Volume 359, Number 5, May 2007, Pages 2155-2189

S 0002-9947(06)04172-9

Article electronically published on December 20, 2006

\title{
SMALL DEFORMATIONS OF POLYGONS AND POLYHEDRA
}

\author{
JEAN-MARC SCHLENKER
}

\begin{abstract}
We describe the first-order variations of the angles of Euclidean, spherical or hyperbolic polygons under infinitesimal deformations such that the lengths of the edges do not change. Using this description, we introduce a vector-valued quadratic invariant $b$ on the space of those isometric deformations which, for convex polygons, has a remarkable positivity property.

We give two geometric applications. The first is an isoperimetric statement for hyperbolic polygons: Among the convex hyperbolic polygons with given edge lengths, there is a unique polygon with vertices on a circle, a horocycle, or on one connected component of the space of points at constant distance from a geodesic, and it has maximal area. The second application is a rigidity result for equivariant polyhedral surfaces in the Minkowski space.
\end{abstract}

RÉSumÉ. On décrit les déformations infinitésimales des angles d'un polygone euclidien, sphérique ou hyperbolique sous les déformations infinitésimales qui préservent les longueurs des arêtes. On en déduit la définition d'un invariant quadratique à valeurs vectorielles $b$ sur l'espace de ces déformations isométriques qui, pour les polygones convexes, a une propriété remarquable de positivité.

On donne deux applications géométriques. La première est un énoncé isoperimétrique pour les polygones hyperboliques: Parmi les polygones hyperboliques convexes dont les longueurs des arêtes sont données, il existe un unique élément dont les sommets sont sur un cercle, un horocycle, ou dans une composante connexe de l'ensemble des points à distance constante d'une géodésique, et son aire est maximale. La seconde application est un résultat de rigidité pour les surfaces polyèdrales équivariantes dans l'espace de Minkowski.

\section{INTRODUCTION}

Moduli spaces of polygons. In this paper, a polygon with $n$ vertices in the Euclidean plane $E^{2}$ (resp. the sphere $S^{2}$, the hyperbolic plane $H^{2}$ or the de Sitter plane $S_{1}^{2}$ ) is a finite sequence of points $v_{1}, v_{2}, \cdots, v_{n}$ in $E^{2}$ (resp. $S^{2}, H^{2}, S_{1}^{2}$ ), with $v_{0}:=v_{n}$, and with $v_{i} \neq v_{i+1}$ for all $i \in\{1, \cdots, n\}$. We call $\mathcal{P}_{n, E}$ (resp. $\mathcal{P}_{n, S}$, $\left.\mathcal{P}_{n, H}, \mathcal{P}_{n, d S}\right)$ the moduli space of polygons in each of the spaces, i.e. the space of polygons quotiented by the group of orientation-preserving isometries of the space. In the sphere and the de Sitter plane, we only consider polygons such that two consecutive vertices are never antipodal (in the sphere, they are at distance less than $\pi$ ) although those cases could be included at the cost of a little more care.

The edge lengths of a polygon are the numbers $l_{1}, \cdots, l_{n}$, where $l_{i}$ is the distance between $v_{i}$ and $v_{i+1}$. Given $l=\left(l_{1}, \cdots, l_{n}\right), \mathcal{P}_{E}(l)$ is the subset of $\mathcal{P}_{n, E}$ of Euclidean

Received by the editors March 8, 2005.

2000 Mathematics Subject Classification. Primary 53C45, 53C50, 51M16.

(C)2006 American Mathematical Society Reverts to public domain 28 years from publication 
polygons with edge lengths equal to $l_{1}, \cdots, l_{n}$. Similar notations will be used for spherical, hyperbolic or de Sitter polygons.

First-order deformations of polygons. Let $p=\left(v_{1}, \cdots, v_{n}\right)$ be a Euclidean polygon, and let $\alpha_{1}, \cdots, \alpha_{n}$ be its angles. An isometric first-order deformation of $p$ is a set of vectors $\dot{v}_{1}, \cdots, \dot{v}_{n}$, with $\dot{v}_{i} \in T_{v_{i}} E^{2}$, such that, if one deforms $p$ infinitesimally by moving each $v_{i}$ along $\dot{v}_{i}$, the lengths of the edges do not change (at first order). This is equivalent to the fact that, for each $i \in\{0,1, \cdots, n-1\}$, $\left\langle v_{i+1}-v_{i}, \dot{v}_{i+1}-\dot{v}_{i}\right\rangle=0$. An infinitesimal first-order deformation of $p$ is trivial if there exists a Killing field $\kappa$ on $\mathbb{R}^{2}$ such that, for each $i, \dot{v}_{i}=\kappa\left(v_{i}\right)$. We mostly consider the isometric first-order deformations of $p$ up to the trivial deformations; for a "generic" polygon $p$, they are canonically associated to the elements of $T_{[p]} \mathcal{P}_{E}(l)$, where $l=\left(l_{1}, \cdots, l_{n}\right)$ is the set of lengths of $p$ and $[p]$ is the image of $p$ under the projection from the space of polygons in $\mathbb{R}^{2}$ of given edge lengths to the quotient under the action of $\operatorname{Isom}\left(\mathbb{R}^{2}\right)$.

Clearly, the isometric first-order deformations of $p$ are determined by the firstorder variations $\dot{\alpha}_{i}$ of its angles. However not all possible variations are possible. We first give some constraints on the possible first-order variations of the angles of polygons in the spaces we consider.

Proposition 1.1. Let $p=\left(v_{1}, \cdots, v_{n}\right)$ be a Euclidean polygon. Let $\alpha_{1}, \cdots, \alpha_{n}$ be its angles, and let $\dot{\alpha}_{1}, \cdots, \dot{\alpha}_{n} \in \mathbb{R}$. Suppose that there exists a first-order isometric deformation of $p$ such that the induced first-order variation of the angles of $p$ is $\left(\dot{\alpha}_{1}, \cdots, \dot{\alpha}_{n}\right)$. Then:

- $\sum_{i=1}^{n} \dot{\alpha}_{i}=0$.

- $\sum_{i=1}^{n} \dot{\alpha}_{i} v_{i}=0$.

Conversely, if those two conditions are satisfied and the vertices of $p$ are not all on a line, then there exists an isometric first-order deformation of $p$ such that the induced first-order variation of the $\alpha_{i}$ are the $\dot{\alpha}_{i}$, and it is unique up to the addition of a trivial deformation.

An analogous but even simpler statement holds for spherical polygons; see e.g. Glu75.

Theorem $\mathbf{A}_{S}$. Let $p=\left(v_{1}, \cdots, v_{n}\right)$ be a spherical polygon. Let $\alpha_{1}, \cdots, \alpha_{n}$ be its angles, and let $\dot{\alpha}_{1}, \cdots, \dot{\alpha}_{n} \in \mathbb{R}$ be a first-order variation of its angles induced by an isometric first-order deformation of $p$. Then:

$$
\sum_{i=1}^{n} \dot{\alpha}_{i} v_{i}=0
$$

where the $v_{i}$ are considered as points in $S^{2} \subset \mathbb{R}^{3}$.

Conversely, if this equation is satisfied by an $n$-uple $\left(\dot{\alpha}_{1}, \cdots, \dot{\alpha}_{n}\right)$ and moreover the $v_{i}$ are not all on a great circle, then there exists an isometric first-order deformation of $p$ such that the $\dot{\alpha}_{i}$ are the associated first-order variations of the $\alpha_{i}$.

Each first-order isometric deformation of $p$ is uniquely determined, up to the addition of a trivial deformation, by the corresponding variation of its angles. Moreover, the space of isometric first-order deformations of $p$ (up to the trivial ones) has dimension at least $n-3$, since a deformation of $p$ is determined by $2 n$ parameters (the positions of the vertices), with $n$ constraints (the lengths of the edges) and a 
group of isometries of dimensions 3 . Therefore, the space of first-order variations of the $\alpha_{i}$, induced by isometric first-order deformations of $p$, always has dimension at least $n-3$. It follows that, unless the $v_{i}$ are on a great circle, the space of isometric first-order deformations of $p$ has dimension $n-3$. We will see at the beginning of Section 2 that this implies the fact, proved by Kapovich and Millson KM99, that in this case the space of polygons with the same edge lengths as $p$ is locally a smooth manifold.

The same description holds in the hyperbolic plane.

Theorem $\mathbf{A}_{H}$. Let $p=\left(v_{1}, \cdots, v_{n}\right)$ be a hyperbolic polygon. Let $\alpha_{1}, \cdots, \alpha_{n}$ be its angles, and let $\dot{\alpha}_{1}, \cdots, \dot{\alpha}_{n} \in \mathbb{R}$ be a first-order variation of its angles induced by an isometric first-order deformation of $p$. Then

$$
\sum_{i=1}^{n} \dot{\alpha}_{i} v_{i}=0
$$

where the $v_{i}$ are considered as points in $H^{2} \subset \mathbb{R}_{1}^{3}$.

Conversely, if this equation is satisfied by an $n$-uple $\left(\dot{\alpha}_{1}, \cdots, \dot{\alpha}_{n}\right)$ and moreover the $v_{i}$ are not all on a hyperbolic geodesic, then there exists an isometric first-order deformation of $p$ such that the $\dot{\alpha}_{i}$ are the associated first-order variations of the $\alpha_{i}$. form:

Here $\mathbb{R}_{1}^{3}$ is the Minkowski 3-dimensional space, i.e. it is $\mathbb{R}^{3}$ with the bilinear

$$
\left\langle(x, y, z),\left(x^{\prime}, y^{\prime}, z^{\prime}\right)\right\rangle=x x^{\prime}+y y^{\prime}-z z^{\prime} .
$$

$H^{2}$ has an isometric embedding in $\mathbb{R}_{1}^{3}$ as a quadric:

$$
H^{2}=\left\{(x, y, z) \in \mathbb{R}_{1}^{3} \mid\langle(x, y, z),(x, y, z)\rangle=-1 \text { and } z>0\right\} .
$$

A quadratic form on first-order deformations. This description of the firstorder deformations of the angles of polygons opens the door to the definition of a quadratic invariant of first-order isometric deformations.

Definition 1.2. Let $p=\left(v_{1}, \cdots, v_{n}\right)$ be a Euclidean polygon, with edge lengths $l_{1}, \cdots, l_{n}$. Let $l:=\left(l_{1}, \cdots, l_{n}\right)$, and let $U \in T_{[p]} \mathcal{P}_{E}(l)$, where $[p]$ is the projection of $p$ in $\mathcal{P}_{E}(l)$. Let $U^{\prime}$ be a first-order deformation of $p$ projecting, under the quotient by the trivial deformations, to $U$. We denote

$$
b(U):=\sum_{i=1}^{n} d \alpha_{i}\left(U^{\prime}\right) d v_{i}\left(U^{\prime}\right),
$$

where the $d \alpha_{i}\left(U^{\prime}\right)$ and the $d v_{i}\left(U^{\prime}\right)$ are the first-order variations of the $\alpha_{i}$ and of the $v_{i}$, respectively, under $U^{\prime}$. Then $b(U)$ does not depend on the choice of $U^{\prime}$.

As a first-order deformation of $p$ defined up to the trivial deformations, $U$ does not define uniquely the first-order variations of the $v_{i}$. However, any choice $U^{\prime}$ of a representative $\left(\dot{v}_{1}, \cdots, \dot{v}_{n}\right)$ will do. Indeed, let $U^{\prime \prime}$ be another first-order deformation of $p$ corresponding to $U$; then $U^{\prime}-U^{\prime \prime}$ is a trivial deformation, so the corresponding variation of the $v_{i}$ is given by $\kappa\left(v_{i}\right)$, where $\kappa$ is a Killing field. But any Killing field $\kappa$ in $E^{2}$ is of the form

$$
\kappa(x)=i \theta x+\tau,
$$


for some $\theta \in \mathbb{R}$ and some vector $\tau \in E^{2}$ (this expression uses the usual identification of $E^{2}$ with $\mathbb{C}$ ). It follows that

$$
\begin{array}{r}
\sum_{i=1}^{n} d \alpha_{i}\left(U^{\prime}\right) d v_{i}\left(U^{\prime}\right)-\sum_{i=1}^{n} d \alpha_{i}\left(U^{\prime \prime}\right) d v_{i}\left(U^{\prime \prime}\right)=\sum_{i=1}^{n} d \alpha_{i}\left(U^{\prime}\right) \kappa\left(v_{i}\right) \\
=\sum_{i=1}^{n} d \alpha_{i}\left(U^{\prime}\right)\left(i \theta v_{i}+\tau\right)=i \theta\left(\sum_{i=1}^{n} d \alpha_{i}\left(U^{\prime}\right) v_{i}\right)+\left(\sum_{i=1}^{n} d \alpha_{i}\left(U^{\prime}\right)\right) \tau,
\end{array}
$$

and both terms vanish by Proposition 1.1

A very similar definition can be used in the sphere or the hyperbolic plane.

Definition 1.3. Let $p=\left(v_{1}, \cdots, v_{n}\right)$ be a polygon in the sphere (resp. the hyperbolic plane) with edge lengths $l_{1}, \cdots, l_{n}$. Let $l:=\left(l_{1}, \cdots, l_{n}\right)$ and let $U \in T_{[p]} \mathcal{P}_{S}(l)$ (resp. $\left.T_{[p]} \mathcal{P}_{H}(l)\right)$ where $[p]$ is the projection of $p$ in $\mathcal{P}_{S}(l)$ (resp. $\left.\mathcal{P}_{H}(l)\right)$. Let $U^{\prime}$ be a first-order deformation of $p$ projecting, under the quotient by the trivial deformations, to $U$. We denote

$$
b(U):=\sum_{i=1}^{n} d \alpha_{i}\left(U^{\prime}\right) d v_{i}\left(U^{\prime}\right),
$$

where the $d \alpha_{i}\left(U^{\prime}\right)$ and the $d v_{i}\left(U^{\prime}\right)$ are the first-order variations of the $\alpha_{i}$ and of the $v_{i}$, considered as points in $\mathbb{R}^{3}$ (resp. $\mathbb{R}_{1}^{3}$ ), respectively, under $U^{\prime}$. Then $b(U)$ does not depend on the choice of $U^{\prime}$.

An argument very similar to the one given above shows that $b(U)$ is indeed independent of the precise deformation $U^{\prime}$ of $p$ which is chosen. In the sphere, any Killing field is of the form

$$
\kappa(x)=Y \times x
$$

for some vector $Y \in \mathbb{R}^{3}$. Therefore, if $U^{\prime \prime}$ is another first-order deformation of $p$ projecting to $U$, then $U^{\prime}-U^{\prime \prime}$ corresponds to a Killing field $\kappa$. Then

$$
\begin{array}{r}
\sum_{i=1}^{n} d \alpha_{i}\left(U^{\prime}\right) d v_{i}\left(U^{\prime}\right)-\sum_{i=1}^{n} d \alpha_{i}\left(U^{\prime \prime}\right) d v_{i}\left(U^{\prime \prime}\right)=\sum_{i=1}^{n} d \alpha_{i}\left(U^{\prime}\right) \kappa\left(v_{i}\right) \\
=\sum_{i=1}^{n} d \alpha_{i}\left(U^{\prime}\right) Y \times v_{i}=Y \times\left(\sum_{i=1}^{n} d \alpha_{i}\left(U^{\prime}\right) v_{i}\right)=0
\end{array}
$$

The same argument can be used in the hyperbolic context, using the fact that the Killing fields of $\mathbb{R}_{1}^{3}$ which vanish at the origin (in other terms, the elements of the Lie algebra $s o(2,1))$ are of the form

$$
\kappa(x)=Y \otimes x
$$

where $\otimes$ is the Minkowski analog of the cross product

$$
\left(Y_{1}, Y_{2}, Y_{3}\right) \otimes\left(x_{1}, x_{2}, x_{3}\right)=\left(Y_{2} x_{3}-Y_{3} x_{2}, Y_{3} x_{1}-Y_{1} x_{3},-Y_{1} x_{2}+Y_{2} x_{1}\right) .
$$

In both cases, the fact that $b$ depends only on the equivalence class of $U^{\prime}$ (under the action of trivial deformations) has an "abstract" interpretation. As defined above, for each polygon $p$ in $S^{2}, b$ defines a quadratic form on the space of isometric first-order deformations of $p$, with values in $\mathbb{R}^{3}$. Let $l=\left(l_{1}, \cdots, l_{n}\right)$ be the edge lengths of $p$. Since $b$ behaves "well" under the action of $S O(3)$ on $S^{2}, b$ actually defines a quadratic form on $T_{[p]} \mathcal{P}_{S}(l)$, where $[p]$ is the projection of $p$ in $\mathcal{P}_{S}(l)$, with values in the vector bundle over $\mathcal{P}_{S}(l)$ which is defined, from the trivial $\mathbb{R}^{3}$-bundle 
over the space of polygons in $S^{2}$ with edge lengths given by $l$, by taking the natural action of $S O(3)$ on both this space of polygons and $\mathbb{R}^{3}$.

A positivity property. The quadratic form $b$ defined above has a striking geometric property when $p$ is a strictly convex spherical or hyperbolic polygon. By a convex polygon, we mean a polygon which is the boundary of a convex domain in $S^{2}$ (resp. $H^{2}$ ), which we call the interior of $p$, and denote by $\operatorname{int}(p)$; a convex polygon is strictly convex if two consecutive edges are never parallel. We state this property first in the spherical setting. Recall that, given a convex spherical polygon $p$, the dual polygon $p^{*}$ is a convex polygon whose interior is the set of points in $S^{2}$ which have positive scalar product with the vertices of $p$ (see e.g. Cox93, Cox57). Each of its vertices is at distance $\pi / 2$ of an edge of $p$, and conversely.

Theorem $\mathbf{B}_{S}$. Let $p$ be a strictly convex polygon in $S^{2}$, and let $U$ be a non-trivial infinitesimal first-order deformation of $p$. Then $b(U) \in\left(\mathbb{R}_{+} \backslash\{0\}\right)$ int $\left(p^{*}\right)$, i.e. $b(u)$ is contained in the positive cone over the interior of $p^{*}$.

There is a geometric interpretation to this property. The space of polygons with given edge lengths $l=\left(l_{1}, \cdots, l_{n}\right), \mathcal{P}_{S}(l)$, has a natural map $\phi$ to $\mathbb{R}^{n}$, where $\phi(p)$ is the family of the angles of $p$. By Theorem $\mathrm{A}_{S}$, the image is locally a submanifold except when $p$ has all its vertices on a great circle. We will see in Section 3 that $b$ is strongly related to the second fundamental form of this submanifold, so that Theorem $\mathrm{B}_{S}$ translates as a convexity property: If $p$ is a convex polygon, the image of $\phi$, in the neighborhood of $\phi(p)$, has a second fundamental form which is positive definite in some directions. Theorem $\mathrm{B}_{S}$ is related to a result of Volkov [Vol56] on (non-infinitesimal) isometric deformations of spherical polygons, and also to a paper of Milka [Mil65.

The same result holds in the hyperbolic or the de Sitter setting. Now the dual of a hyperbolic polygon is a convex, space-like de Sitter polygon, and conversely; both can be defined as in the sphere, using the Minkowski metric on $\mathbb{R}_{1}^{3}$ (see e.g. Cox43, Cox57, where the "polarities" corresponding to the duality used here have a very central role, and also [Riv86, RH93] where the duality was used in a very interesting way).

Theorem $\mathbf{B}_{H}$. Let $p$ be a strictly convex polygon in $H^{2}$ and let $U$ be a non-trivial infinitesimal first-order deformation of $P$. Then $b(U) \in\left(\mathbb{R}_{+} \backslash\{0\}\right) \operatorname{int}\left(p^{*}\right)$, i.e. $b(U)$ is in the positive cone over the interior of $p^{*}$.

An isoperimetric statement for hyperbolic polygons. The first application of Theorems $\mathrm{A}_{H}$ and $\mathrm{B}_{H}$ that we consider is to an isoperimetric problem for hyperbolic polygons. The next theorem is the hyperbolic analog of a statement which was proved by Steiner [Ste42] in the spherical setting, but was known earlier in the Euclidean case (see $\mathrm{Sie02}$ for recent progress on more elaborate statements even in the Euclidean case).

Given $l=\left(l_{1}, \cdots, l_{n}\right)$, we call $\mathcal{P}_{S}^{c}(l)$ the space of convex polygons in $S^{2}$ with edge lengths equal to the $l_{i}$, considered up to global isometries in $S^{2}$, and $\mathcal{P}_{H}^{c}(l)$ the space of convex hyperbolic polygons with edge lengths equal to the $l_{i}$, again considered up to global isometries.

Theorem C. Let $l=\left(l_{1}, \cdots, l_{n}\right)$ be such that $\mathcal{P}_{H}^{c}(l)$ is not empty; then there exists a unique element $p \in \mathcal{P}_{H}^{c}(l)$ which has its vertices either on a circle, on a horocycle, 
or on a connected set of points at fixed distance from a geodesic. Moreover, $p$ has maximal area.

There is a similar statement, which is slightly simpler, in the Euclidean plane and in the sphere; in both cases, there is a unique polygon of maximal area (among the polygons with fixed edge lengths) and it has its vertices on a circle. The proof given here for hyperbolic polygons also works in the spherical setting.

The first point of the proof, which is given in Section 4, is that, as a direct consequence of Theorem $\mathrm{A}_{H}$, a hyperbolic polygon is a critical point of the area, restricted to polygons with the same edge lengths, if and only if its vertices are either on a circle, a horocycle, or a connected component of the space of points at fixed distance from a geodesic. Theorem $\mathrm{B}_{S}$ (resp. $\mathrm{B}_{H}$ ) also has an interesting meaning in this context: It implies that the area, as a function defined on the space of convex polygons with given edge lengths, is "often" strictly concave for a natural metric.

The second point of the proof is that, on the boundary of the space of convex polygons with given edge lengths, the interior normal derivative of the area is positive. It follows that there exists at least one local maximum of the area in the interior. But a direct argument shows that, given the edge lengths, there is at most one polygon with its vertices on a circle, a horocycle, or on one connected component of the set of points at fixed distance from a geodesic. It follows that there is exactly one such polygon, and that it is the unique maximum of the area.

The infinitesimal rigidity of convex polyhedra. The positivity property of $b$ in the spherical setting leads to a simple proof of the infinitesimal rigidity of convex polyhedra in the Euclidean space. Although the first proof was given by Dehn [Deh16], the statement follows from the ideas of Legendre [LegII] and Cauchy Cau13, as explained by Sabitov in Sab04. Other proofs have been given, in particular, by Kann Kan90] and Filliman [Fil92].

Theorem 1.4 (Legendre, Cauchy, Dehn). Let $P$ be a convex polyhedron in $\mathbb{R}^{3}$. Any first-order deformation of $P$ which does not change its combinatorics or the metrics on its faces is trivial, i.e. induced by a global Killing field.

The proof given here is in Section 5. It is quite close to a proof given by Milka Mil65, and bears some relations with a proof of the global rigidity of convex Euclidean polyhedra discovered by Pogorelov [Pog56]. It is given here mostly as a motivation for the proof of Theorem 6.2

Fuchsian polyhedral surfaces in the Minkowski space. The techniques described here have a natural application to another rigidity problem, concerning polyhedral surfaces in the Minkowski 3-dimensional space. We consider space-like polyhedral surfaces; rather than closed polyhedra, which can be defined as images of convex polyhedral maps from the sphere, we consider equivariant polyhedra, which are the images of a polyhedral map from the universal cover of a surface of genus at least 2 which is equivariant (more precise definitions can be found in Section 6). We will prove that, among such surfaces, those which are "Fuchsian" - the associated representation from the fundamental group of the surface to the isometry group of $\mathbb{R}_{1}^{3}$ has its image in $S O(2,1)$ - are infinitesimally rigid, i.e. any first-order deformation of those surfaces which does not change the induced metric is trivial; this is Theorem 6.2 
There is an analogous result for smooth, equivariant, Fuchsian, convex surfaces LS00, which was proved by related methods. Actually, finding a polyhedral version of the results of [LS00] was the main motivation for the present work. This question was also studied by I. Iskhakov [Isk00], who provided some partial results. There are some natural motivations for this question in a broader geometric context; see e.g. Sch03

One feature of the proofs of Theorem 1.4 and of Theorem 6.2 is the existence of a distinguished point, which appears quite artificial in the Euclidean context; for surfaces in the Minkowski space, however, it is quite natural and even necessary, since the point which is fixed by the representation of the fundamental group already plays a special role.

The infinitesimal rigidity of Fuchsian equivariant surfaces in the Minkowski space is equivalent, thanks to the "Pogorelov map" used e.g. in [LS00, to similar rigidity statements in the de Sitter or anti-de Sitter space, so that polyhedral rigidity results in those spaces could be obtained as a consequence of the rigidity theorem for polyhedral surfaces in $\mathbb{R}_{1}^{3}$ proved here. However, another proof of the rigidity of Fuchsian equivariant surfaces in the de Sitter or the anti-de Sitter space has recently been developed by F. Fillastre (in preparation), and it applies in particular in the polyhedral setting. So it should also be possible to prove the result stated hereon polyhedral Fuchsian surfaces in $\mathbb{R}_{1}^{3}$-from the statements obtained by Fillastre in the de Sitter or the anti-de Sitter space.

To prove Theorem 6.2 we will follow the proof of Theorem 1.4 but the polygons that will be considered will be in the de Sitter plane rather than in the sphere. Recall that, in addition to the hyperbolic plane, $\mathbb{R}_{1}^{3}$ contains another quadric, the de Sitter plane $S_{1}^{2}$, which is a complete, constant curvature 2-dimensional Lorentz manifold (see e.g. Cox43, Cox57, O’N83]):

$$
S_{1}^{2}=\left\{(x, y, z) \in \mathbb{R}_{1}^{3} \mid\langle(x, y, z),(x, y, z)\rangle=1\right\} .
$$

Polygons in the de Sitter plane can be defined as in the sphere; the edges can be of different types, either space-like, light-like or time-like. The notion of angle is more subtle than in the sphere (see e.g. Sch98, Sch01, Ale03), and it is quite natural to consider the angles of a polygon as complex numbers, with real part either $0, \pm \pi / 2$ or $\pi$. A key point is that, with those definitions and the corresponding definitions for the lengths of the edges, the main triangle formulas in the sphere remain valid in the de Sitter plane, a simple fact which we prove in Section 6 for completeness.

The first-order deformations of polygons in the de Sitter plane, in terms of the first-order variation of the angles, can be described as in the sphere or the hyperbolic plane.

Theorem $\mathbf{A}_{d S}$. Let $p=\left(v_{1}, \cdots, v_{n}\right)$ be a polygon in the de Sitter plane. Let $\alpha_{1}, \cdots, \alpha_{n}$ be its angles, and let $\dot{\alpha}_{1}, \cdots, \dot{\alpha}_{n} \in \mathbb{R}$ be a first-order variation of its angles induced by an isometric first-order deformation of $p$. Then

$$
\sum_{i=1}^{n} \dot{\alpha}_{i} v_{i}=0
$$

where the $v_{i}$ are considered as points in $\mathbb{R}_{1}^{3}$.

Conversely, if this equation is satisfied by an $n$-uple $\left(\dot{\alpha}_{1}, \cdots, \dot{\alpha}_{n}\right)$ and moreover the $v_{i}$ are not all on geodesic, then there exists an isometric first-order deformation of $p$ such that the $\dot{\alpha}_{i}$ are the associated first-order variations of the $\alpha_{i}$. 
In this context, the quadratic form $b$ can be defined as in the sphere. It has the same positivity property for strictly convex, space-like polygons (a notion which is defined here with some care; see Section 6). The notion of polygon dual to a space-like, convex polygon is defined in Section 6 as in the sphere, it is a polygon in the hyperbolic plane. The analog of Theorem $\mathrm{B}_{S}$ also holds, with a coefficient $i$ coming from the way we define the angles in the de Sitter plane.

Theorem $\mathbf{B}_{d S}$. Let $p$ be a convex, space-like polygon in $S_{1}^{2}$ and let $U$ be an infinitesimal first-order deformation of $P$. Then $i b(U) \in\left(\mathbb{R}_{+} \backslash\{0\}\right)$ int $\left(p^{*}\right)$, i.e. $i b(U)$ is contained in the positive cone over the interior of the dual polygon $p^{*}$.

The argument used in the proof of Theorem 1.4, translated to the Minkowski setting, yields a rigidity statement for Fuchsian polyhedral surfaces in $\mathbb{R}_{1}^{3}$, Theorem 6.2 .

Metrics on moduli spaces. One consequence of Theorem $\mathrm{B}_{S}$ is that it shows that the invariant $b$ can be used to define some natural metrics on the moduli space of convex polygons in $S^{2}$ (and similarly in the hyperbolic plane) 1 .

Notation. Throughout this paper, we set $\mathbb{R}_{+}^{*}:=\mathbb{R}_{+} \backslash\{0\}$.

\section{Deformations of POLYGONS}

The moduli space of polygons. The geometry of the moduli space of polygons has been studied rather extensively, in particular by Kapovich and Millson (see e.g. KM95, KM99,). We recall here only some very elementary properties which should clarify parts of the proofs below. We consider here polygons in the plane, however all the comments in this paragraph hold also for spherical, hyperbolic or de Sitter polygons, with some obvious adaptations.

Let $n \geq 3$, let $P_{n, E}$ be the space of polygons with $n$ vertices in $\mathbb{R}^{2}$. Recall that a polygon is a sequence of vertices $v_{1}, \cdots, v_{n}=v_{0}$ such that, for each $i$, $v_{i} \neq v_{i+1}$. The isometry group $\operatorname{Isom}\left(\mathbb{R}^{2}\right)$ of $\mathbb{R}^{2}$ acts without fixed points on $P_{n, E}$, so we consider the quotient $\mathcal{P}_{n, E}=P_{n, E} / \operatorname{Isom}\left(\mathbb{R}^{2}\right) . \mathcal{P}_{n, E}$ is a smooth manifold of dimension $2 n-3$, which can also be considered as a smooth algebraic variety.

There is a family of $n$ functions naturally defined on $\mathcal{P}_{n, E}$; if $p \in P_{n, E}$ is a polygon, with $p=\left(v_{1}, \cdots, v_{n}\right)$, then

$$
\lambda_{i}(p):=d\left(v_{i}, v_{i+1}\right) .
$$

Those $n$ functions are clearly invariant under the action of $\operatorname{Isom}\left(\mathbb{R}^{2}\right)$, so they define natural functions on $\mathcal{P}_{n, E}$, which we still call $\lambda_{i}$. Note that it would be algebraically more natural to consider the squares of the distances, but we stick to the more natural definition from an elementary geometry viewpoint (for spherical polygons one could consider the cosine of the distance, and for hyperbolic polygons the cosh).

Now let $\left(l_{1}, \cdots, l_{n}\right)$ be an $n$-uple of positive numbers, recall that $\mathcal{P}_{E}(l)$ is the (moduli) space of polygons with edge lengths $l_{i}$. So

$$
\mathcal{P}_{E}(l):=\left\{p \in \mathcal{P}_{n, E} \mid \forall i \in\{1, \cdots, n\}, \lambda_{i}(p)=l_{i}\right\},
$$

\footnotetext{
${ }^{1} \mathrm{~A}$ reader interested in this question may check version 2 of this text [Sch04], which is more detailed on this point.
} 
so that $\mathcal{P}_{E}(l)$ is an algebraic subvariety of $\mathcal{P}_{n, E}$. Let $p \in \mathcal{P}_{E}(l)$, consider the space of its first-order infinitesimal deformations:

$$
\mathcal{T}_{p}:=\operatorname{ker}\left(d \lambda_{1}\right) \cap \cdots \cap \operatorname{ker}\left(d \lambda_{n}\right) \subset T_{p} \mathcal{P}_{n, E} .
$$

Clearly $\mathcal{T}_{p}$ has dimension at least $n-3$, since it is defined by $n$ equations. If those $n$ equations are linearly independent, then, by the inverse function theorem, in the neighborhood of $p, \mathcal{P}_{E}(l)$ is a smooth submanifold of $\mathcal{P}_{n, E}$ (and also a smooth algebraic subvariety) of dimension $n-3$.

Euclidean polygons. We first indicate the proof of Proposition 1.1 The simplest proof is perhaps obtained by taking a limit, in Theorem $\mathrm{A}_{S}$ below, when the lengths of the edges go to 0 . It is however possible to give a simpler direct proof (this was pointed out by Sergiu Moroianu).

Let $p=\left(v_{1}, \cdots, v_{n}\right)$ be a Euclidean polygon, with edge lengths $l_{1}, \cdots, l_{n}$, so that, for each $i \in\{1, \cdots, n\}, l_{i}=\left\|v_{i+1}-v_{i}\right\| \neq 0$ (with $v_{n}=v_{0}$ ). For each $i$, let $\theta_{i}$ be the (oriented) angle between the oriented $x$-axis and $v_{i+1}-v_{i}$. The possible values of the angles $\theta_{1}, \cdots, \theta_{n}$, in the neighborhood of $p$, are defined by the condition that $\sum_{i=1}^{n} l_{i} e^{i \theta_{i}}=0$, because $v_{n+1}=v_{1}$.

In an isometric first-order deformation of $p$, it follows that

$$
\sum_{i=1}^{n} \dot{\theta}_{i} l_{i} e^{i \theta_{i}}=0
$$

Suppose (adding a trivial deformation if necessary) that, in the deformation of $p$ we consider, the direction of $v_{1}-v_{0}$ does not vary. Then, for each $i, \dot{\theta}_{i}=\sum_{j=1}^{i} \dot{\alpha}_{j}$. Therefore,

$$
0=\sum_{i=1}^{n} l_{i} e^{i \theta_{i}} \sum_{j=1}^{i} \dot{\alpha}_{i}=\sum_{j=1}^{n} \dot{\alpha}_{j} \sum_{i=j}^{n} l_{i} e^{i \theta_{i}}
$$

We can also suppose - still without loss of generality- that $v_{0}=0$. Then, for each $j$

$$
v_{j}=\sum_{i=0}^{j-1} l_{i} e^{i \theta_{i}}=-\sum_{i=j}^{n} l_{i} e^{i \theta_{i}},
$$

and it follows that

$$
\sum_{j=1}^{n} \dot{\alpha}_{j} v_{j}=0 .
$$

In addition, it is well known that, for any Euclidean polygon

$$
\sum_{i=1}^{n} \alpha_{i}=2 \pi k
$$

where $k$ is the winding number of the polygon. It follows that, in any first-order deformation, $\sum_{i=1}^{n} \dot{\alpha}_{i}=0$.

The two conditions in the statement of Proposition 1.1 are linearly independent if and only if the $v_{i}$ are not all on a line. When the $v_{i}$ are not collinear, the vector space of $n$-uples $\left(\dot{\alpha}_{1}, \cdots, \dot{\alpha}_{i}\right)$ satisfying them has dimension $n-3$. Therefore, the arguments in the previous paragraph show that this space has dimension exactly $n-3$, and the possible first-order variations of the $\alpha_{i}$ are exactly the solutions of the two equations in the statement of the proposition. 
Spherical polygons. We now prove Theorem $\mathrm{A}_{S}$. A direct proof is possible 2 , but the proof given here is geometric and much simpler.

Let $p=\left(v_{1}, \cdots, v_{n}\right)$ be a spherical polygon, and let $U$ be a first-order isometric deformation of $p$. Consider the polyhedral cone $C$ over $p$, i.e. the union of the halflines with endpoint at 0 which intersect $p \subset S^{2} \subset \mathbb{R}^{3}$. $C$ has $n$ faces $f_{1}, \cdots, f_{n}$, with $f_{i}$ equal to the cone over the edge $e_{i}$ of $p$. Since $\alpha_{i}$ is the angle between the edges $e_{i}$ and $e_{i+1}$ of $p$, it is also equal to the dihedral angle between $f_{i}$ and $f_{i+1}$.

The first-order deformation $U$ of $P$ determines a first-order deformation $V$ of $C$. For each $i \in\{1, \cdots, n\}, V$ acts on each face of $C$ as a Killing field, i.e. there exist vectors $u_{i}$ and $y_{i}$ such that $V$ acts on $f_{i}$ as the vector field $\kappa_{i}$ defined by

$$
\forall x \in \mathbb{R}^{3}, \kappa_{i}(x)=u_{i}+y_{i} \times x .
$$

Then $\kappa_{i+1}-\kappa_{i}=0$ on the intersection of $f_{i}$ and $f_{i+1}$, and the difference between $\kappa_{i}$ and $\kappa_{i+1}$ is equal to an infinitesimal rotation of angle $d \alpha_{i}(U)$ and axis $f_{i} \cap f_{i+1}$. It follows that

$$
y_{i+1}=y_{i}+d \alpha_{i}(U) v_{i}
$$

and Theorem $\mathrm{A}_{S}$ follows since the sum of the differences $y_{i+1}-y_{i}$, for $i$ going from 1 to $n$, vanishes.

Hyperbolic polygons. The same argument can be used to prove Theorem $\mathrm{A}_{H}$. $C$ is now a polyhedral cone in the Minkowski space $\mathbb{R}_{1}^{3}$, and its faces are time-like. The restriction of the infinitesimal deformation $V$ to each face $f_{i}$ is a Killing vector, which is now of the form $\kappa_{i}(x)=u_{i}+y_{i} \otimes x$. The proof then proceeds as for the sphere.

\section{A positivity Result}

In this section we prove Theorem $\mathrm{B}_{S}$, as well as its hyperbolic analog Theorem $\mathrm{B}_{H}$, after some preliminary computations concerning the first-order deformations of quadrilaterals.

Deformations of quadrilaterals. Here the quadrilateral $q=\left(v_{1}, v_{2}, v_{3}, v_{4}\right)$ is considered, for which no 3 of the vertices are collinear, and set $v_{0}:=v_{4}$. We use the notations apparent in Figure 1, in particular $t$ is the distance between $v_{1}$ and $v_{3}$. We also call $d_{i, j}$ the distance between $v_{i}$ and $v_{j}$.

Consider a first-order isometric deformation of $q$ and suppose for instance that $v_{0}$ and $v_{1}$ are fixed (this can be achieved by adding a trivial deformation). Then $v_{2}$ moves in the direction of the circle of center $v_{1}$ containing it. Since $v_{1}, v_{2}$ and $v_{3}$ are not collinear, the circle of center $v_{3}$ containing $v_{2}$ is not tangent to the circle of center $v_{1}$ containing $v_{2}$, so that any first-order displacement of $v_{2}$ induces a non-zero first-order variation of the distance from $v_{2}$ to $v_{3}$.

The same argument shows that any first-order displacement of $v_{3}$, preserving its distance to $v_{0}$, induces a non-zero first-order variation of its distance to $v_{2}$. It follows that there is a 1-dimensional vector space of isometric first-order deformations of $q$ (up to the trivial deformations), they are parametrized for instance by $t$, the distance from $v_{1}$ to $v_{3}$. So we consider the first-order deformation $U$ of $q$ such that $d t(U)=1$.

\footnotetext{
${ }^{2}$ One such proof is available in the first version of this paper, see http://front. math.ucdavis.edu/math.DG/0410058 and then choose "v1".
} 


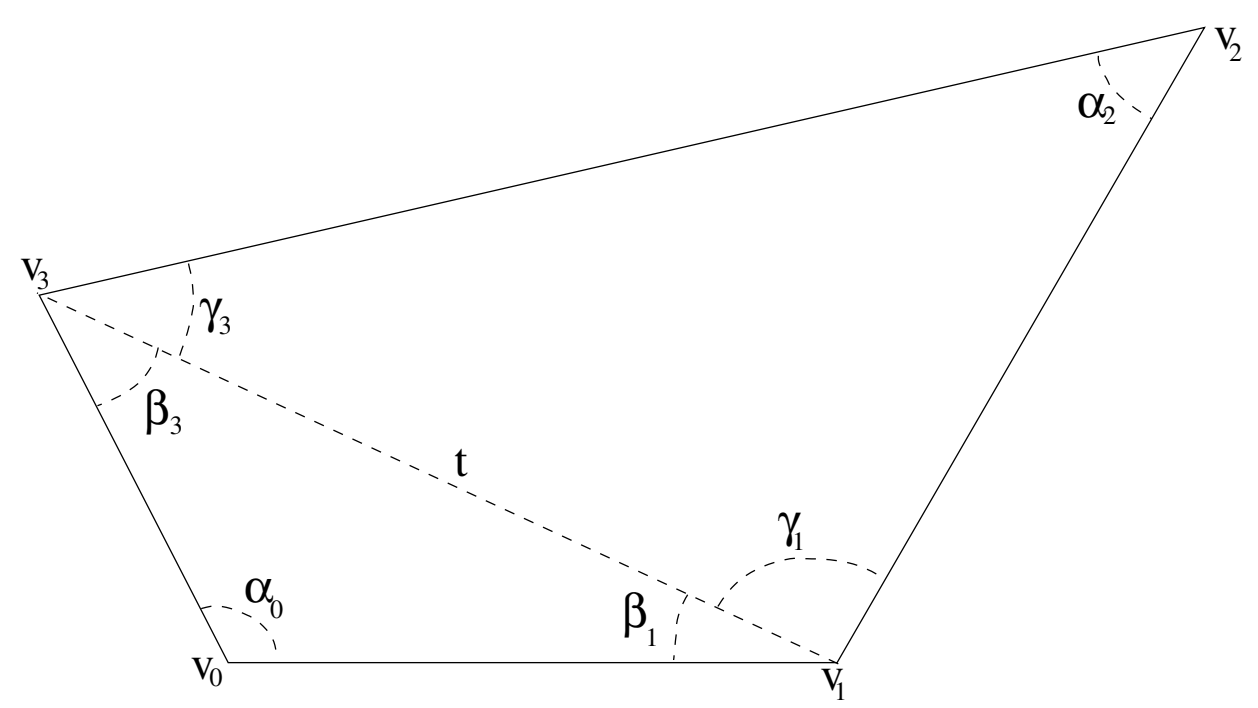

FiguRE 1. Deformation of a non-degenerate quadrilateral

A well-known spherical geometry formula states that

$$
\cos (t)=\cos \left(d_{01}\right) \cos \left(d_{03}\right)+\cos \left(\alpha_{0}\right) \sin \left(d_{01}\right) \sin \left(d_{03}\right),
$$

so that

$$
d \alpha_{0}(U)=\frac{\sin (t)}{\sin \left(d_{01}\right) \sin \left(d_{03}\right) \sin \left(\alpha_{0}\right)} .
$$

The same computation (or a symmetry argument) shows that

$$
d \alpha_{2}(U)=\frac{\sin (t)}{\sin \left(d_{12}\right) \sin \left(d_{23}\right) \sin \left(\alpha_{2}\right)} .
$$

Moreover, equation (3.1), applied to the triangle $\left(v_{0}, v_{1}, v_{3}\right)$, yields

$$
\cos \left(\beta_{1}\right)=\frac{\cos \left(d_{03}\right)-\cos \left(d_{01}\right) \cos (t)}{\sin \left(d_{01}\right) \sin (t)},
$$

so that

$$
\begin{aligned}
-\sin \left(\beta_{1}\right) d \beta_{1}(U) & =\frac{\cos \left(d_{01}\right) \sin ^{2}(t)-\left(\cos \left(d_{03}\right)-\cos \left(d_{01}\right) \cos (t)\right) \cos (t)}{\sin \left(d_{01}\right) \sin ^{2}(t)} \\
& =\frac{\cos \left(d_{01}\right)-\cos \left(d_{03}\right) \cos (t)}{\sin \left(d_{01}\right) \sin ^{2}(t)}
\end{aligned}
$$

But, by the sine formula for spherical triangles

$$
\frac{\sin \left(\beta_{1}\right)}{\sin \left(d_{03}\right)}=\frac{\sin \left(\alpha_{0}\right)}{\sin (t)}
$$

so that

$$
d \beta_{1}(U)=\frac{\cos \left(d_{03}\right) \cos (t)-\cos \left(d_{01}\right)}{\sin \left(d_{01}\right) \sin \left(d_{03}\right) \sin (t) \sin \left(\alpha_{0}\right)}
$$


The same computation (or a symmetry argument) shows that

$$
d \gamma_{1}(U)=\frac{\cos \left(d_{23}\right) \cos (t)-\cos \left(d_{12}\right)}{\sin \left(d_{12}\right) \sin \left(d_{23}\right) \sin (t) \sin \left(\alpha_{2}\right)}
$$

and, taking the sum, we obtain

$$
d \alpha_{1}(U)=\frac{\cos \left(d_{03}\right) \cos (t)-\cos \left(d_{01}\right)}{\sin \left(d_{01}\right) \sin \left(d_{03}\right) \sin (t) \sin \left(\alpha_{0}\right)}+\frac{\cos \left(d_{23}\right) \cos (t)-\cos \left(d_{12}\right)}{\sin \left(d_{12}\right) \sin \left(d_{23}\right) \sin (t) \sin \left(\alpha_{2}\right)}
$$

By the same computation (or a symmetry argument)

$$
d \alpha_{3}(U)=\frac{\cos \left(d_{01}\right) \cos (t)-\cos \left(d_{03}\right)}{\sin \left(d_{01}\right) \sin \left(d_{03}\right) \sin (t) \sin \left(\alpha_{0}\right)}+\frac{\cos \left(d_{12}\right) \cos (t)-\cos \left(d_{23}\right)}{\sin \left(d_{12}\right) \sin \left(d_{23}\right) \sin (t) \sin \left(\alpha_{2}\right)}
$$

Proof of Theorem $\mathbf{B}_{S}$. Let $p=\left(v_{1}, \cdots, v_{n}\right)$ be a strictly convex spherical polygon. Recall that, by Definition 1.2, for any first-order deformation $U$ of $p$, defined up to the Killing fields, we have

$$
b(U)=\sum_{i=0}^{n} d \alpha_{i}\left(U^{\prime}\right) d v_{i}\left(U^{\prime}\right),
$$

where $U^{\prime}$ is any representative of $U$, i.e. any first-order deformation of $p$ corresponding to $U$ under the quotient by the trivial deformations.

So $b$ is a quadratic form on $T_{p} \mathcal{P}_{S}(l)$, where $l=\left(l_{1}, \cdots, l_{n}\right)$ is the $n$-uple of the edge lengths of $p$. It is natural to define a bilinear form associated to $b$, which we call $b_{2}$; it is defined as follows: If $U_{1}^{\prime}$ and $U_{2}^{\prime}$ are two first-order deformations of $p$, then

$$
b_{2}\left(U_{1}^{\prime}, U_{2}^{\prime}\right):=\frac{1}{2}\left(\sum_{i=1}^{n} d \alpha_{i}\left(U_{1}^{\prime}\right) d v_{i}\left(U_{2}^{\prime}\right)+\sum_{i=1}^{n} d \alpha_{i}\left(U_{2}^{\prime}\right) d v_{i}\left(U_{1}^{\prime}\right)\right) .
$$

As for $b$, an important point is that if one adds a Killing field to either $U_{1}^{\prime}$ or $U_{2}^{\prime}$, the result does not change. Moreover, it will be useful below to note that each of the two sums in the definition of $b_{2}$ is invariant under this transformation. Indeed, if $Y_{1}, Y_{2} \in \mathbb{R}^{3}$ are two vectors, let $V_{1}, V_{2}$ be the trivial deformations defined by $d v_{i}\left(V_{1}\right)=Y_{1} \times v_{i}, d v_{i}\left(V_{2}\right)=Y_{2} \times v_{i}$; then, for each $i \in\{1, \cdots, n\}, d \alpha_{i}\left(U_{1}^{\prime}+V_{1}\right)=$ $d \alpha_{i}\left(U_{i}^{\prime}\right)$, and

$$
\begin{aligned}
\sum_{i=1}^{n} d \alpha_{i}\left(U_{1}^{\prime}+V_{1}\right) d v_{i}\left(U_{2}^{\prime}+V_{2}\right) & =\sum_{i=1}^{n} d \alpha_{i}\left(U_{1}^{\prime}\right)\left(d v_{i}\left(U_{2}^{\prime}\right)+Y_{2} \times v_{i}\right) \\
& =\sum_{i=1}^{n} d \alpha_{i}\left(U_{1}^{\prime}\right) d v_{i}\left(U_{2}^{\prime}\right)+Y_{2} \times \sum_{i=1}^{n} d \alpha_{i}\left(U_{1}^{\prime}\right) v_{i} \\
& =\sum_{i=1}^{n} d \alpha_{i}\left(U_{1}^{\prime}\right) d v_{i}\left(U_{2}^{\prime}\right)
\end{aligned}
$$

and the same computation can be applied to the second sum in the definition of $b_{2}$.

Lemma 3.1. Let $p=\left(v_{1}, \cdots, v_{n}=v_{0}\right)$ be a polygon such that no 3 vertices are collinear. Let $U$ be an isometric first-order deformation of $p$, such that $v_{1}$ and $v_{2}$ are fixed. There exists a unique decomposition

$$
U=U_{2}+U_{3}+\cdots+U_{n-2}
$$

such that, for all $i \in\{2, \cdots, n-2\}, U_{i}$ vanishes on $v_{1}, v_{2}, \cdots, v_{i}$, and acts on $v_{i+1}, \cdots, v_{n-1}, v_{n}=v_{0}$ as a rigid motion. 
Proof. We define a sequence of isometric first-order deformations of $p$ recursively, as follows. First note that, since $v_{0}, v_{1}$ and $v_{3}$ are not collinear, there is a unique first-order isometric deformation $V_{2}$ of the quadrilateral $\left(v_{0}, v_{1}, v_{2}, v_{3}\right)$ such that $v_{1}$ and $v_{2}$ are fixed, and that the first-order displacement of $v_{3}$ is the same for $V_{2}$ and for $U$. Then define a first-order deformation $U_{2}$ as follows:

- $v_{1}$ and $v_{2}$ are fixed.

- $v_{3}$ moves under $U_{2}$ as under $V_{2}$, i.e. as under $U$.

- The restriction of $U_{2}$ to $v_{3}, v_{4}, \cdots, v_{n}=v_{0}$ is equal to the restriction to those vertices of a Killing field, i.e. the corresponding part of $p$ moves in a "rigid" way.

It is possible to define such a deformation because, at first order, the distance between $v_{3}$ and $v_{0}$ does not vary under the deformation $V_{2}$.

Now consider the first-order deformation $U-U_{2}$ of $p$. It is isometric - as the difference of two isometric deformations - and it vanishes at $v_{1}, v_{2}$ and $v_{3}$. This shows, because $v_{1}, v_{4}$ and $v_{0}$ are not collinear, that there exists a unique isometric first-order deformation $V_{3}$ of the quadrilateral $\left(v_{1}, v_{3}, v_{4}, v_{0}\right)$ which vanishes at $v_{1}$ and $v_{3}$ and acts on $v_{4}$ as $U-U_{2}$. Let $U_{3}$ be the unique first-order deformation of $p$ which vanishes at $v_{1}, v_{2}$ and $v_{3}$, and which acts on $v_{4}, \cdots, v_{n-1}, v_{0}$ as a Killing field (i.e. "rigidly").

Now $U-U_{2}-U_{3}$ is an isometric first-order deformation of $p$ which vanishes at $v_{1}, v_{2}, v_{3}$ and $v_{4}$, and we can iterate this construction until we obtain a decomposition

$$
U=U_{2}+\cdots+U_{n-2},
$$

which has the property described in the lemma.

Consider now a first-order deformation $U$ of $p$. Since $p$ is strictly convex, it is nondegenerate in the sense of Lemma 3.1. Applying this lemma yields a decomposition $U=U_{2}+\cdots+U_{n-2}$ such that, for all $i \in\{2, \cdots, n-2\}, U_{i}$ vanishes on $v_{1}, v_{2}, \cdots, v_{i}$, and acts on $v_{i+1}, \cdots, v_{n-1}, v_{n}=v_{0}$ as a rigid motion.

Lemma 3.2. For each $i, j \in\{2, \cdots, n-2\}$ with $i \neq j,\left\langle b_{2}\left(U_{i}, U_{j}\right), v_{1}\right\rangle=0$. Therefore,

$$
\left\langle b(U), v_{1}\right\rangle=\left\langle\sum_{i=1}^{n} b\left(U_{i}\right), v_{1}\right\rangle .
$$

Proof. Suppose (without loss of generality) that $i<j$. For each $i \in\{2, \cdots, n-2\}$, we add a Killing field to $U_{i}$ to obtain a first-order deformation $U_{i}^{\prime}$ which acts on $v_{1}, v_{2}, \cdots, v_{i}$ as a rigid motion, and vanishes on $v_{i+1}, \cdots, v_{n-1}, v_{n}=v_{0}$. It follows from the remark above that

$$
b_{2}\left(U_{i}, U_{j}\right)=\frac{1}{2}\left(\sum_{k=1}^{n} d \alpha_{k}\left(U_{i}\right) d v_{k}\left(U_{j}\right)+\sum_{k=1}^{n} d \alpha_{k}\left(U_{j}^{\prime}\right) d v_{k}\left(U_{i}^{\prime}\right)\right) .
$$

Note that $d \alpha_{k}\left(U_{i}\right)$ vanishes for all values of $k$ except $0,1, i$ and $i+1$. But $d v_{k}\left(U_{j}\right)$ vanishes, by definition of $U_{j}$, for $k=1, \cdots, j$. Symmetrically, $d \alpha_{k}\left(U_{j}^{\prime}\right)$ vanishes except for $k=0,1, j, j+1$, while $d v_{k}\left(U_{i}^{\prime}\right)$ vanishes for $k=i+1, \cdots, n$, so that

$$
b_{2}\left(U_{i}, U_{j}\right)=\frac{1}{2}\left(d \alpha_{0}\left(U_{i}\right) d v_{0}\left(U_{j}\right)+d \alpha_{1}\left(U_{j}^{\prime}\right) d v_{1}\left(U_{i}^{\prime}\right)\right) .
$$


Since $U_{j}$ is an isometric first-order deformation, we have

$$
0=d\left\langle v_{0}, v_{1}\right\rangle\left(U_{j}\right)=\left\langle d v_{0}\left(U_{j}\right), v_{1}\right\rangle+\left\langle v_{0}, d v_{1}\left(U_{j}\right)\right\rangle .
$$

But $d v_{1}\left(U_{j}\right)=0$ by definition of $U_{j}$, so that $\left\langle d v_{0}\left(U_{j}\right), v_{1}\right\rangle=0$. Since $d v_{1}\left(U_{i}^{\prime}\right) \in$ $T_{v_{1}} S^{2},\left\langle v_{1}, d v_{1}\left(U_{i}^{\prime}\right)\right\rangle=0$, and it follows that $\left\langle b_{2}\left(U_{i}, U_{j}\right), v_{1}\right\rangle=0$.

The value of $\left\langle b\left(U_{i}\right), v_{1}\right\rangle$ is given by the next lemma.

Lemma 3.3. Let $q:=\left(v_{1}, v_{i}, v_{i+1}, v_{0}\right)$ and let $\alpha_{1}^{\prime}, \alpha_{i}^{\prime}, \alpha_{i+1}^{\prime}, \alpha_{0}^{\prime}$ be its angles. Let $V$ be the first-order isometric deformation of $q$ which vanishes on $v_{1}$ and $v_{i}$ and under which the distance between $v_{1}$ and $v_{i+1}$ varies at speed 1 . Then, if $d_{j, k}:=d\left(v_{j}, v_{k}\right)$, we have

$$
\left\langle v_{1}, d \alpha_{i+1}^{\prime}(V) d v_{i+1}(V)+d \alpha_{0}^{\prime}(V) d v_{0}(V)\right\rangle=\frac{\sin ^{2}\left(d_{1, i+1}\right) \sin \left(\alpha_{1}^{\prime}\right)}{\sin \left(d_{0, i+1}\right) \sin \left(d_{i, i+1}\right) \sin \left(\alpha_{0}^{\prime}\right) \sin \left(\alpha_{i}^{\prime}\right)} .
$$

Proof. We have already noted that $\left\langle v_{1}, d v_{0}(V)\right\rangle=0$ because $V$ does not change, at first order, the distance between $v_{0}$ and $v_{1}$. So the only non-vanishing term is the one involving $\left\langle v_{1}, d v_{i+1}(V)\right\rangle$, and

$$
\left\langle v_{1}, d v_{i+1}(V)\right\rangle=d\left\langle v_{1}, v_{i+1}\right\rangle(V)=d \cos \left(d_{1, i+1}\right)(V)=-\sin \left(d_{1, i+1}\right) .
$$

But $d \alpha_{i+1}(V)$ is given by equation (3.5); we now have slightly different notation: $U$ is replaced by $V$, the indices 2 (resp. 3) by $i$ (resp. $i+1$ ). So,

$$
d \alpha_{i+1}^{\prime}(V)=\frac{\cos \left(d_{0,1}\right) \cos (t)-\cos \left(d_{0, i+1}\right)}{\sin \left(d_{0,1}\right) \sin \left(d_{0, i+1}\right) \sin (t) \sin \left(\alpha_{0}^{\prime}\right)}+\frac{\cos \left(d_{1, i}\right) \cos (t)-\cos \left(d_{i, i+1}\right)}{\sin \left(d_{1, i}\right) \sin \left(d_{i, i+1}\right) \sin (t) \sin \left(\alpha_{i}^{\prime}\right)},
$$

where $t:=d_{1, i+1}$. Following the notations of Figure 1 , we call $\beta_{1}^{\prime}$ and $\beta_{i+1}^{\prime}$ the angles at $v_{1}$ and $v_{i+1}$, respectively, of the triangle $\left(v_{0}, v_{1}, v_{i+1}\right)$, and we call $\gamma_{1}^{\prime}$ and $\gamma_{i+1}^{\prime}$ the angles at $v_{1}$ and $v_{i+1}$, respectively, of the triangle $\left(v_{1}, v_{i}, v_{i+1}\right)$. Using equation (3.1), we get

$$
\begin{aligned}
d \alpha_{i+1}^{\prime}(V) & =-\frac{\cos \left(\beta_{1}^{\prime}\right) \sin \left(d_{0,1}\right) \sin (t)}{\sin \left(d_{0,1}\right) \sin \left(d_{0, i+1}\right) \sin (t) \sin \left(\alpha_{0}^{\prime}\right)}-\frac{\cos \left(\gamma_{1}^{\prime}\right) \sin \left(d_{1, i}\right) \sin (t)}{\sin \left(d_{1, i}\right) \sin \left(d_{i, i+1}\right) \sin (t) \sin \left(\alpha_{i}^{\prime}\right)} \\
& =-\sin (t)\left(\frac{\cos \left(\beta_{1}^{\prime}\right)}{\sin \left(d_{0, i+1}\right) \sin \left(\alpha_{0}^{\prime}\right) \sin (t)}+\frac{\cos \left(\gamma_{1}^{\prime}\right)}{\sin \left(d_{i, i+1}\right) \sin \left(\alpha_{i}^{\prime}\right) \sin (t)}\right) \\
& =-\frac{\sin (t)}{\sin \left(d_{0, i+1}\right) \sin \left(d_{i, i+1}\right) \sin \left(\alpha_{0}^{\prime}\right) \sin \left(\alpha_{i}^{\prime}\right)} \\
& \times\left(\cos \left(\beta_{1}^{\prime}\right) \frac{\sin \left(d_{i, i+1}\right) \sin \left(\alpha_{i}^{\prime}\right)}{\sin (t)}+\cos \left(\gamma_{1}^{\prime}\right) \frac{\sin \left(d_{0, i+1}\right) \sin \left(\alpha_{0}^{\prime}\right)}{\sin (t)}\right) .
\end{aligned}
$$

But, by the sine law for spherical triangles,

$$
\frac{\sin \left(\alpha_{i}^{\prime}\right)}{\sin (t)}=\frac{\sin \left(\gamma_{1}^{\prime}\right)}{\sin \left(d_{i, i+1}\right)}, \frac{\sin \left(\alpha_{0}^{\prime}\right)}{\sin (t)}=\frac{\sin \left(\beta_{1}^{\prime}\right)}{\sin \left(d_{0, i+1}\right)},
$$

so that

$$
\begin{aligned}
d \alpha_{i+1}^{\prime}(V) & =-\sin (t) \frac{\cos \left(\beta_{1}^{\prime}\right) \sin \left(\gamma_{1}^{\prime}\right)+\cos \left(\gamma_{1}^{\prime}\right) \sin \left(\beta_{1}^{\prime}\right)}{\sin \left(d_{0, i+1}\right) \sin \left(d_{i, i+1}\right) \sin \left(\alpha_{0}^{\prime}\right) \sin \left(\alpha_{i}^{\prime}\right)} \\
& =-\sin (t) \frac{\sin \left(\alpha_{1}^{\prime}\right)}{\sin \left(d_{0, i+1}\right) \sin \left(d_{i, i+1}\right) \sin \left(\alpha_{0}^{\prime}\right) \sin \left(\alpha_{i}^{\prime}\right)},
\end{aligned}
$$

and the result follows. 
It follows directly from Lemma 3.2 and from Lemma 3.3 that $\left\langle b(U), v_{1}\right\rangle$ is a sum of positive terms, so it is positive. By symmetry the same holds for all the other vertices. Since $b(U)$ has positive scalar product with all the vertices of $p$, it is contained in the positive cone over the interior of the dual polygon $p^{*}$, and this proves Theorem $\mathrm{B}_{S}$.

Proof of Theorem $\mathbf{B}_{H}$. The same proof applies in the hyperbolic case, with some small differences in the computations. We only state the hyperbolic analog of Lemma 3.3 .

Lemma 3.4. Let $q:=\left(v_{1}, v_{i}, v_{i+1}, v_{0}\right)$ be a quadrilateral in the hyperbolic plane, and let $\alpha_{1}^{\prime}, \alpha_{i}^{\prime}, \alpha_{i+1}^{\prime}, \alpha_{0}^{\prime}$ be its angles. Let $V$ be the first-order isometric deformation of $q$ which vanishes on $v_{1}$ and $v_{i}$ and under which the distance between $v_{1}$ and $v_{i+1}$ varies at speed 1 . Then, if $d_{j, k}:=d\left(v_{j}, v_{k}\right)$, we have

$\left\langle v_{1}, d \alpha_{i+1}^{\prime}(V) d v_{i+1}(V)+d \alpha_{0}^{\prime}(V) d v_{0}(V)\right\rangle=\frac{\sinh ^{2}\left(d_{1, i+1}\right) \sin \left(\alpha_{1}^{\prime}\right)}{\sinh \left(d_{0, i+1}\right) \sinh \left(d_{i, i+1}\right) \sin \left(\alpha_{0}^{\prime}\right) \sin \left(\alpha_{i}^{\prime}\right)}$.

The proof is based on some computations which are quite parallel to those made above for first-order deformations of spherical quadrilaterals, but now we discuss hyperbolic quadrilaterals. We use again the notations apparent in Figure 11 The basic triangle formula is now

$$
\cosh (t)=\cosh \left(d_{0,1}\right) \cosh \left(d_{0, i+1}\right)-\cos \left(\alpha_{0}^{\prime}\right) \sinh \left(d_{0,1}\right) \sinh \left(d_{0, i+1}\right),
$$

from which it follows that, in a first-order deformation $U$ such that $t$ varies at speed 1 , we have

$$
d \alpha_{0}^{\prime}(U)=\frac{\sinh (t)}{\sin \left(\alpha_{0}\right) \sinh \left(d_{0,1}\right) \sinh \left(d_{0, i+1}\right)},
$$

and the same computation shows that

$$
d \alpha_{i}^{\prime}(U)=\frac{\sinh (t)}{\sin \left(\alpha_{i}^{\prime}\right) \sinh \left(d_{1, i}\right) \sinh \left(d_{i, i+1}\right)} .
$$

Moreover, equation (3.7) yields

$$
\cos \left(\beta_{1}\right)=\frac{-\cosh \left(d_{0, i+1}\right)+\cosh \left(d_{0,1}\right) \cosh (t)}{\sinh \left(d_{0,1}\right) \sinh (t)},
$$

so that

$$
\begin{aligned}
-\sin & \left(\beta_{1}\right) d \beta_{1}(U) \\
& =\frac{\cosh \left(d_{0,1}\right) \sinh ^{2}(t)-\left(-\cosh \left(d_{0, i+1}\right)+\cosh \left(d_{0,1}\right) \cosh (t)\right) \cosh (t)}{\sinh \left(d_{0,1}\right) \sinh ^{2}(t)} \\
& =\frac{-\cosh \left(d_{0,1}\right)+\cosh \left(d_{0, i+1}\right) \cosh (t)}{\sinh \left(d_{0,1}\right) \sinh ^{2}(t)} .
\end{aligned}
$$

But

so that

$$
\frac{\sin \left(\beta_{1}\right)}{\sinh \left(d_{0, i+1}\right)}=\frac{\sin \left(\alpha_{0}^{\prime}\right)}{\sinh (t)}
$$

$$
d \beta_{1}(U)=\frac{-\cosh \left(d_{0, i+1}\right) \cosh (t)+\cosh \left(d_{0,1}\right)}{\sinh \left(d_{0,1}\right) \sinh \left(d_{0, i+1}\right) \sinh (t) \sin \left(\alpha_{0}^{\prime}\right)} .
$$


By symmetry,

$$
d \gamma_{1}(U)=\frac{-\cosh \left(d_{i, i+1}\right) \cosh (t)+\cosh \left(d_{1, i}\right)}{\sinh \left(d_{1, i}\right) \sinh \left(d_{i, i+1}\right) \sinh (t) \sin \left(\alpha_{i}^{\prime}\right)},
$$

and taking the sum

$$
\begin{aligned}
d \alpha_{1}^{\prime}(U)= & \frac{-\cosh \left(d_{0, i+1}\right) \cosh (t)+\cosh \left(d_{0,1}\right)}{\sinh \left(d_{0,1}\right) \sinh \left(d_{0, i+1}\right) \sinh (t) \sin \left(\alpha_{0}^{\prime}\right)} \\
& +\frac{-\cosh \left(d_{i, i+1}\right) \cosh (t)+\cosh \left(d_{1, i}\right)}{\sinh \left(d_{1, i}\right) \sinh \left(d_{i, i+1}\right) \sinh (t) \sin \left(\alpha_{i}^{\prime}\right)} .
\end{aligned}
$$

By the same computation (or a symmetry argument)

$$
\begin{aligned}
d \alpha_{i+1}^{\prime}(U)= & \frac{-\cosh \left(d_{0,1}\right) \cosh (t)+\cosh \left(d_{0, i+1}\right)}{\sinh \left(d_{0,1}\right) \sinh \left(d_{0, i+1}\right) \sinh (t) \sin \left(\alpha_{0}^{\prime}\right)} \\
& +\frac{-\cosh \left(d_{1, i}\right) \cosh (t)+\cosh \left(d_{i, i+1}\right)}{\sinh \left(d_{1, i}\right) \sinh \left(d_{i, i+1}\right) \sinh (t) \sin \left(\alpha_{i}^{\prime}\right)} .
\end{aligned}
$$

The end of the proof then proceeds as in the spherical case, we leave the details to the interested reader.

The submanifold of angles for given edge lengths. One possible way to interpret geometrically the positivity property of $b$ for spherical polygons is in terms of a kind of convexity property of a submanifold of codimension 3 in $\mathbb{R}^{n}$, defined as the set of possible angles of convex polygons with given edge lengths. This submanifold is often smooth, and when it has singularities, they are located at precise points. Its tangent space at each point is given by Theorem $\mathrm{A}_{S}$, while Theorem $\mathrm{B}_{S}$ indicates that its second fundamental form is always on "one side", as explained below.

We consider here a spherical polygon $p$ with $n$ vertices, and call $l=\left(l_{1}, \cdots, l_{n}\right)$ the family of its edge length. Then we call $\mathcal{A}_{S}(l)$ the space of $n$-uples $\left(\alpha_{1}, \cdots, \alpha_{n}\right)$ such that there exists a polygon $p^{\prime} \in \mathcal{P}_{S}(l)$ with angles equal to the $\alpha_{i}$. There is a natural map $\Phi_{l}$ from $\mathcal{P}_{S}(l)$ into $(0,2 \pi)^{n}$, with image $\mathcal{A}_{S}(l)$, which is injective because a spherical polygon is entirely defined - up to global isometries in $S^{2}$-by its edge lengths and its angles.

Theorem $\mathrm{A}_{S}$, along with the remarks at the beginning of Section 2, show that $\mathcal{A}_{S}(l)$ is locally a smooth submanifold of codimension 3 of $(\mathbb{R} / 2 \pi \mathbb{Z})^{n}$, except when all the vertices of the polygon lie on a spherical geodesic. Clearly, this is possible only when there exists $\left(\epsilon_{1}, \cdots, \epsilon_{n}\right) \in\{-1,1\}$ and $k \in \mathbb{Z}$ such that

$$
\sum_{i=1}^{n} \epsilon_{i} l_{i}=2 k \pi
$$

Then the singular points of $\mathcal{A}_{S}(l)$ can happen only at the points of $(\mathbb{R} / 2 \pi \mathbb{Z})^{n}$ which have all their coordinates equal to 0 or $\pi$.

Let $q \in P_{S}(l)$, with vertices $v_{1}, \cdots, v_{n}$. Let $\alpha_{1}, \cdots, \alpha_{n}$ be the angles of $q$, we suppose that they are not all equal to 0 or $\pi$, so that $\mathcal{A}_{S}(l)$ is locally smooth in the neighborhood of $\alpha:=\left(\alpha_{1}, \cdots, \alpha_{n}\right)$. The tangent space of $\mathcal{A}_{S}(l)$ is described by Theorem $\mathrm{A}_{S}$. More precisely, there is a natural isomorphism between $\mathbb{R}^{3}$ and the normal space of $\mathcal{A}_{S}(l)$ at $\alpha$, defined as follows:

$$
\forall w \in \mathbb{R}^{3}, \Psi_{q}(w):=\left(\left\langle v_{1}, w\right\rangle, \cdots,\left\langle v_{n}, w\right\rangle\right) .
$$


Theorem $\mathrm{A}_{S}$ shows that $\Psi_{q}\left(\mathbb{R}^{3}\right)$ is the orthogonal complement to the tangent space of $\mathcal{A}_{S}(l)$ at $\alpha$, i.e. the normal space of $\mathcal{A}_{S}(l)$ at $\alpha$.

Now $b$ appears naturally as the second fundamental form of $\mathcal{A}_{S}(l)$.

Remark 3.5. Let $U \in T_{q} P_{S}(l)$ be a first-order isometric deformation of $q$ and let $w \in \mathbb{R}^{3}$. Then

$$
\left\langle I I\left(d \Phi_{l}(U), d \Phi_{l}(U)\right), \Psi_{q}(w)\right\rangle=-\langle b(U), w\rangle,
$$

where $I I$ is the second fundamental form of $\mathcal{A}_{S}(l)$.

Proof. Let $w \in \mathbb{R}^{3}$. Call $\nabla$ the flat connection on $\mathbb{R}^{n}$. By definition of the second fundamental form of $\mathcal{A}_{S}(l)$, we have

$$
\begin{aligned}
\left\langle I I\left(d \Phi_{l}(U), d \Phi_{l}(U)\right), \Psi_{q}(w)\right\rangle= & -\left\langle\nabla_{d \Phi_{l}(U)} \Psi_{q}(w), d \Phi_{l}(U)\right\rangle \\
= & -\left\langle\left(\left\langle d v_{i}(U), w\right\rangle\right)_{i=1}^{n},\left(d \alpha_{i}(U)\right)_{i=1}^{n}\right\rangle \\
= & -\sum_{i=1}^{n}\left\langle d \alpha_{i}(U) d v_{i}(U), w\right\rangle \\
= & -\langle b(U), w\rangle .
\end{aligned}
$$

There is a direct translation of Theorem $\mathrm{B}_{S}$ in this context, indicating a kind of convexity property of $\mathcal{A}_{S}(l)$ at the points which are the images of strictly convex polygons.

Proposition 3.6. Suppose that $q$ is strictly convex. Let $Q^{*}$ be the polyhedral cone in $N_{\Phi_{l}(q)} \mathcal{A}_{S}(l)$ which is dual to the cone $\Psi_{q}\left(\left(\mathbb{R}_{+}^{*}\right) q\right)$, for the metric induced on $N_{q} \mathcal{A}_{S}(l)$ by the metric on $\mathbb{R}^{n}$. Then

$$
\forall V \in T_{\Phi_{l}(q)} \mathcal{A}_{S}(l), I I(V, V) \in-Q^{*}
$$

Proof. By the previous remark and Theorem $\mathrm{B}_{S}$,

$$
\forall U \in T_{q} P_{S}(l), \forall w \in \mathbb{R}_{+}^{*} q,\left\langle I I\left(d \Phi_{l}(U), d \Phi_{l}(U)\right), \Psi_{q}(w)\right\rangle<0,
$$

so that

$$
\forall V \in T_{\Phi_{l}(q)} \mathcal{A}_{S}(l), \forall W \in \Psi_{q}\left(\mathbb{R}_{+}^{*} q\right),\langle I I(V, V), W\rangle<0,
$$

and the result follows.

The same considerations hold also for hyperbolic polygons, based on Theorem $\mathrm{A}_{H}$ for the description of the tangent space, and on Theorem $\mathrm{B}_{H}$ for the convexity property of the submanifold of the angles of polygons with given edge lengths.

In the Euclidean case, one can again consider the space $\mathcal{A}_{E}(l)$ of angles of polygons with edge lengths given by $l$. Then $\mathcal{A}_{E}(l)$ is a codimension 2 submanifold of the hyperplane of $\sum_{i=1}^{n} \alpha_{i}=0$. However, it does not appear to have any obvious "convexity" property.

\section{ISOPERIMETRIC PROBLEMS FOR SPHERICAL OR HYPERBOLIC POLYGONS}

The theorems given above-Theorem $\mathrm{A}_{S}$ and Theorem $\mathrm{B}_{S}$ for spherical polygons, Theorem $\mathrm{A}_{H}$ and Theorem $\mathrm{B}_{H}$ for hyperbolic polygons - have very simple applications to isoperimetric questions in the two settings. The spherical result was apparently discovered by Steiner [Ste42], and is analog to a Euclidean statement which was known earlier (see e.g. [Sie02] for recent progress on related but more elaborate Euclidean statements). The proofs are strongly related to Theorems $\mathrm{A}_{S}$ and $\mathrm{B}_{S}$ (resp. $\mathrm{A}_{H}$ and $\left.\mathrm{B}_{H}\right)$. 
Critical points of the area functional. First, Theorem $\mathrm{A}_{S}$ and Theorem $\mathrm{A}_{H}$ lead directly to a description of the critical points of the area function over the space of polygons with given edge lengths.

Lemma 4.1. Let $p$ be a spherical polygon, with edge lengths given by $l=\left(l_{1}, \cdots, l_{n}\right)$. Suppose that the vertices of $p$ are not all on a geodesic. Then $p$ is a critical point of the area in $\mathcal{P}_{S}(l)$ if and only if all vertices of $p$ are on a circle.

Lemma 4.2. Let $p$ be a hyperbolic polygon, with edge lengths given by $l=\left(l_{1}, \cdots, l_{n}\right)$. Suppose that the vertices of $p$ are not all on a geodesic. Then $p$ is a critical point of the area in $\mathcal{P}_{H}(l)$ if and only if all vertices of $p$ are either on a circle, on a horocycle, or on a connected component of the set of points at fixed, non-zero distance from a geodesic.

Note that the polygons are not required to be convex. It follows from the results given below that, for strictly convex polygons, the critical points are actually maxima, but it is probably not the case for non-convex polygons. Both lemmas are proved together.

Proof. By the Gauss-Bonnet formula, $p$ is a critical point of the area functional if and only if, for any first-order isometric deformation of $p$, the sum of the angles of $p$ remains constant. By Theorem $\mathrm{A}_{S}$ (resp. Theorem $\mathrm{A}_{H}$ ) this happens if and only if

$$
\forall \dot{\alpha}_{1}, \cdots, \dot{\alpha}_{n} \in \mathbb{R},\left(\sum_{i=1}^{n} \dot{\alpha}_{i} v_{i}=0 \Rightarrow \sum_{i=1}^{n} \dot{\alpha}_{i}=0\right) .
$$

By an elementary linear algebra argument using the transposed equation, this is true if and only if there exists a vector $u \in \mathbb{R}^{3}$ (resp. $\mathbb{R}_{1}^{3}$ ) such that

$$
\forall i \in\{1, \cdots, n\},\left\langle u, v_{i}\right\rangle=1 \text {, }
$$

which holds if and only if the $v_{i}$ are on an affine plane in $\mathbb{R}^{3}$ (resp. $\mathbb{R}_{1}^{3}$ ) not containing the origin. The result follows, because the intersection of $S^{2}$ with an affine plane in $\mathbb{R}^{3}$ is a circle (or a point) while the intersection of $H^{2}$ with an affine plane in $\mathbb{R}_{1}^{3}$ not containing the origin is either a circle, a horocycle, or a connected component of the set of points at fixed, non-zero distance from a geodesic.

Uniqueness of critical polygons. In light of the previous paragraph, it is interesting to remark that, for a given set of edge lengths, there is at most one strictly convex polygon which is a critical point of the area. We first consider spherical polygons, for which the result has been well known for many years. We include a complete proof here for completeness, and because it will be used in the proof of the hyperbolic result below.

Lemma 4.3. Let $l_{1}, \cdots, l_{n} \in \mathbb{R}_{+}^{*}$. There is at most one convex polygon $p \in \mathcal{P}_{S}^{c}(l)$ which has its vertices on a circle.

Proof. We suppose that $l_{1}$ is the largest of the $l_{i}$.

Suppose that the vertices of $p$ are on a circle of radius $l$ and center $x_{0}$ in $S^{2}$. Then the edge of $p$ of length $l_{i}$ is "seen" from $x_{0}$ under an angle $\theta_{i}$, and the sine law for spherical triangles shows that

$$
\sin \left(\theta_{i} / 2\right)=\frac{\sin \left(l_{i} / 2\right)}{\sin (l)} .
$$


Setting $S_{i}:=\sin \left(l_{i} / 2\right)$ and $S:=\sin (l)$, we have again two possibilities:

- Either $x_{0}$ is in the interior of $p$, and then

$$
\sum_{i=1}^{n} \arcsin \left(S_{i} / S\right)=\pi
$$

- Or $x_{0}$ is not in the interior of $p$, and then

$$
\sum_{i=2}^{n} \arcsin \left(S_{i} / S\right)=\arcsin \left(S_{1} / S\right) .
$$

Moreover, in both cases, we have

$$
\sum_{i=2}^{n} \arcsin \left(S_{i} / S\right)<\pi
$$

and

Set

$$
S_{1} / S=\sin \left(\sum_{i=2}^{n} \arcsin \left(S_{i} / S\right)\right)
$$

$$
F(s):=s \sin \left(\sum_{i=2}^{n} \arcsin \left(\frac{S_{i}}{s}\right)\right) \text {. }
$$

Then

$$
\begin{aligned}
& F^{\prime}(s)=\sin \left(\sum_{i=2}^{n} \arcsin \left(\frac{S_{i}}{s}\right)\right)+s \cos \left(\sum_{i=2}^{n} \arcsin \left(\frac{S_{i}}{s}\right)\right)\left(\sum_{i=2}^{n} \frac{-S_{i} / s^{2}}{\sqrt{1-S_{i}^{2} / s^{2}}}\right) \\
& =\cos \left(\sum_{i=2}^{n} \arcsin \left(\frac{S_{i}}{s}\right)\right)\left[\tan \left(\sum_{i=2}^{n} \arcsin \left(\frac{S_{i}}{s}\right)\right)-\sum_{i=2}^{n} \tan \left(\arcsin \left(\frac{S_{i}}{s}\right)\right)\right] .
\end{aligned}
$$

When $\sum_{i=2}^{n} \arcsin \left(S_{i} / s\right) \in[\pi / 2, \pi)$, both terms in the first equation are nonnegative, and it follows that $F^{\prime}(s)$ is positive. When $\sum_{i=2}^{n} \arcsin \left(S_{i} / s\right) \in(0, \pi / 2)$, the second equation shows the same result, because the cosine is positive, while tan is convex on $(0, \pi / 2)$, so that the second factor is also positive. Therefore, $F$ is increasing, and it follows that there is at most one $s$ such that $F(s)=S_{1}$, and therefore at most one possible value of $S=\sin (l)$.

But, given $l$, the angles $\alpha_{i}$ are uniquely determined by the $l_{i}$, so that there is at most one polygon with vertices on a circle and with edge lengths equal to the $l_{i}$.

We now consider the hyperbolic case. This is a step in the proof of Theorem C.

Lemma 4.4. Let $l_{1}, \cdots, l_{n} \in \mathbb{R}_{+}^{*}$. There is at most one convex polygon $p \in \mathcal{P}_{H}^{c}(l)$ which has its vertices either on a circle, on a horocycle, or on a connected component of the set of points at constant, non-zero distance from a geodesic.

Proof. We suppose again that $l_{1}$ is the largest of the $l_{i}$.

Suppose first that the vertices of $p$ are on a circle of radius $l$ and center $x_{0}$ in $H^{2}$. Then the edge of $p$ of length $l_{i}$ is "seen" from $x_{0}$ under an angle $\theta_{i}$, and the sine law for hyperbolic triangles shows that

$$
\sin \left(\theta_{i} / 2\right)=\frac{\sinh \left(l_{i} / 2\right)}{\sinh (l)} .
$$


Setting $S_{i}:=\sinh \left(l_{i} / 2\right)$ and $S:=\sinh (l)$, we have again two possibilities:

- Either $x_{0}$ is in the interior of $p$, and then

$$
\sum_{i=1}^{n} \arcsin \left(S_{i} / S\right)=\pi \text {. }
$$

- Or $x_{0}$ is not in the interior of $p$, and then

$$
\sum_{i=2}^{n} \arcsin \left(S_{i} / S\right)=\arcsin \left(S_{1} / S\right) .
$$

Moreover, in both cases, we have

$$
S_{1} / S=\sin \left(\sum_{i=2}^{n} \arcsin \left(S_{i} / S\right)\right)
$$

and, since sin is concave on $[0, \pi]$

$$
S_{1} / S<\sum_{i=2}^{n} \sin \left(\arcsin \left(S_{i} / S\right)\right)=\sum_{i=2}^{n} S_{i} / S,
$$

so that

$$
S_{1}<\sum_{i=2}^{n} S_{i}
$$

Suppose now that the vertices of $p$ are on a horocycle $h$. An elementary argument in hyperbolic geometry shows that the distance, along $h$, between the vertices of an edge of length $l_{i}$ is equal to $2 \sinh \left(l_{i} / 2\right)$.

In the projective model of $H^{2}$, the horocycle $h$ appears as an ellipse, tangent to the boundary at infinity at a point $x_{0}$. In this model, $p$ appears as a convex polygon, with vertices on this ellipse. There is exactly one edge $e$ of $p$ such that $x_{0}$ is on one side of $e$, while all the other edges of $p$ are on the other side. Clearly, $e$ has to be the edge of $p$ of largest lengths (because its endpoints are at the largest distance along $h$ ) and, in this case,

$$
S_{1}=\sum_{i=2}^{n} S_{i}
$$

Finally, suppose that all vertices of $p$ are on a connected component $c$ of the set of points at distance $l \neq 0$ from a geodesic $g_{0}$. Let $e_{1}, \cdots, e_{n}$ be the edges of $p$; the sine formula for hyperbolic triangles shows that, if $a_{i}$ is the distance between the orthogonal projections on $g_{0}$ of $e_{i}$ and $e_{i+1}$, then

$$
\sinh \left(a_{i} / 2\right)=\frac{\sinh \left(l_{i} / 2\right)}{\sinh (l)} .
$$

In the projective model of $H^{2}$, the set of points at distance $l$ from $g_{0}$ appears as an ellipse, tangent to the boundary at infinity of $H^{2}$ at the endpoints of $g_{0}$, and $p$ appears as a polygon with vertices on one side of that ellipse (see Figure 2). By convexity, there is one edge $e$ of $p$ such that $g_{0}$ is on one side, and all the other edges of $p$ on the other. This edge has maximal length, and

$$
a_{1}=\sum_{i=2}^{n} a_{i},
$$




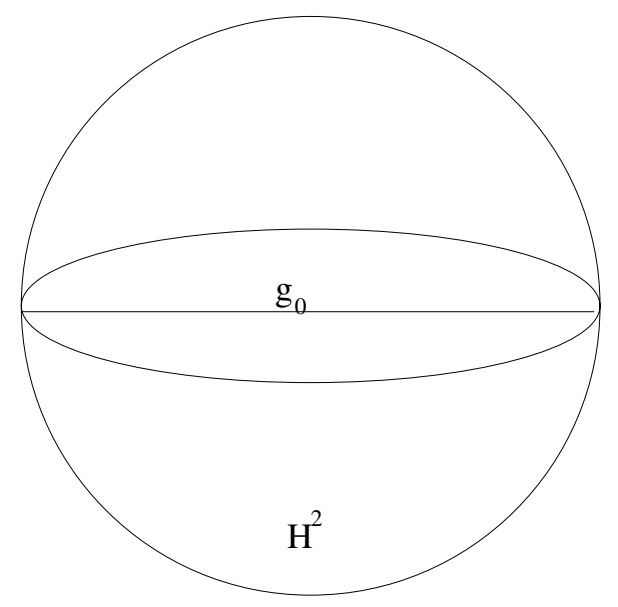

Figure 2. The set of points at constant distance from a geodesic

so that

$$
\operatorname{argsinh}\left(S_{1} / S\right)=\sum_{i=2}^{n} \operatorname{argsinh}\left(S_{i} / S\right)
$$

Then, taking the sinh, we have, because sinh is convex on $(0, \infty)$,

$$
S_{1} / S=\sinh \left(\sum_{i=2}^{n} \operatorname{argsinh}\left(S_{i} / S\right)\right)>\sum_{i=2}^{n} S_{i} / S,
$$

so that $S_{1}>\sum_{i=2}^{n} S_{i}$.

Considering those three cases, it is clear that the $l_{i}$ determine whether there can exist a convex polygon $p$ with edge lengths equal to the $l_{i}$ and vertices on a circle, a horocycle, or a connected component of the set of points at constant, positive distance from a geodesic.

(1) If $S_{1}<\sum_{i=2}^{n} S_{i}$, then the only possibility is that the vertices of $p$ are on a circle.

(2) If $S_{1}=\sum_{i=2}^{n} S_{i}$, then the vertices of $p$ can only be on a horocycle.

(3) If $S_{1}>\sum_{i=2}^{n} S_{i}$, the vertices of $p$ can only be on a connected component of the set of points at constant, positive distance from a geodesic.

In the first case, we proceed as in the proof of Lemma 4.3 above, and set

$$
F(s):=s \sin \left(\sum_{i=2}^{n} \arcsin \left(\frac{S_{i}}{s}\right)\right) .
$$

Either equation (4.1) or equation (4.2) is satisfied; in both cases, $F(S)=S_{1}$. However the same proof as for Lemma 4.3 shows that $F$ is strictly increasing, so that there is at most one possible value of $S$. As in the spherical case, it is easy to check that a polygon with vertices on a circle is uniquely determined (up to global isometries) by its edge lengths and the radius of the circle; this finishes the proof in case (1).

In the second case, the polygon $p$ is clearly uniquely determined (up to global isometries) by the distances between its vertices on the horocycle, so the statement in the lemma holds. 
In case (3), it is necessary to modify slightly the argument used in the spherical case. We set

$$
G(s):=s \sinh \left(\sum_{i=2}^{n} \operatorname{argsinh}\left(\frac{S_{i}}{s}\right)\right) .
$$

Now

$$
\begin{aligned}
& G^{\prime}(s)= \sinh \left(\sum_{i=2}^{n} \operatorname{argsinh}\left(\frac{S_{i}}{s}\right)\right)+s \cosh \left(\sum_{i=2}^{n} \operatorname{argsinh}\left(\frac{S_{i}}{s}\right)\right)\left(\sum_{i=2}^{n} \frac{-S_{i} / s^{2}}{\sqrt{1+S_{i}^{2} / s^{2}}}\right) \\
&=\cosh \left(\sum_{i=2}^{n} \operatorname{argsinh}\left(\frac{S_{i}}{s}\right)\right)\left[\tanh \left(\sum_{i=2}^{n} \operatorname{argsinh}\left(\frac{S_{i}}{s}\right)\right)\right. \\
&\left.-\sum_{i=2}^{n} \tanh \left(\operatorname{argsinh}\left(\frac{S_{i}}{s}\right)\right)\right] .
\end{aligned}
$$

But the first factor in the right-hand term is positive, while the second factor is negative because tanh is concave on $(0, \infty)$. So $G$ is strictly decreasing, and there is at most one value of $s$ such that $G(s)=S_{1}$. It follows that there is at most one polygon with edge lengths equal to the $l_{i}$ and with vertices on a connected component of the set of points at constant, positive distance from a geodesic.

Boundary behavior of the area functional. Another, simple element in the proof of Theorem $\mathrm{C}$ is that, on the boundary of the space $\mathcal{P}_{S}^{c}(l)\left(\operatorname{resp} . \mathcal{P}_{H}^{c}(l)\right)$, the interior derivative of the area is positive.

Lemma 4.5. Let $p \in \partial \mathcal{P}_{S}^{c}(l)$ (resp. $p \in \partial \mathcal{P}_{H}^{c}(l)$ ) be a polygon with at least one angle equal to $\pi$. Then the interior normal derivative of the area at $p$ is positive.

Proof. Let $v_{1}, \cdots, v_{n}$ be the vertices of $p$, and let $I \subset\{1, \cdots, n\}$ be the set of all $i \in\{1, \cdots, n\}$ at which the angle is equal to $\pi$.

We define an isometric first-order deformation $U$ of $p$ as follows. $U$ vanishes at all vertices of $p$ where the angle is strictly less than $\pi$. For each maximal segment $s$ contained in $p$, of length $l>0$, we choose a positive strictly concave function $f_{s}$ on $[0, l]$. If $p \subset S^{2}$, we also require that $f^{\prime \prime}<-f$ on $[0, l]$; this is possible because, since $p$ is convex, its length is less than $2 \pi$, and $l<\pi$.

Suppose that the vertices of $p$ contained in $s$ are $v_{i}, v_{i+1}, \cdots, v_{j}$, so that $\{i, \cdots, j\}$ $\subset I$. Then we choose the first-order deformation $U$ at $v_{k}$, for all $k \in\{i, \cdots, j\}$, as the vector normal to $s$, towards the exterior of $p$, of norm equal to $f\left(t_{k}\right)$, where $t_{k}$ is the coordinate of $v_{k}$ along $s$ (from either of the endpoints of $s$ ).

Clearly, $U$ is an isometric deformation, it is towards the interior of $\mathcal{P}_{S}^{c}(l)$ (resp. of $\left.\mathcal{P}_{H}^{c}(l)\right)$, and the first-order variation of the area under $U$ is positive. The result follows.

Proof of Theorem C. We can now give the proof of both Theorem $\mathrm{C}$ and the corresponding, classical corresponding result in the sphere.

By Lemma 4.5 the area has at least one local maximum in each connected component of $\mathcal{P}_{S}^{c}(l)$ (resp. of $\mathcal{P}_{H}^{c}(l)$ ). By Lemma 4.2, each critical point of the area is a polygon with vertices on a circle, a horocycle, or a connected component of the set of points at constant distance from a geodesic. By Lemma 4.4, there is at most one such critical point. So the area has exactly one critical point in $\mathcal{P}_{H}^{c}(l)$, which is a maximum. By the way, it also follows that $\mathcal{P}_{H}^{c}(l)$ is connected. 
Note that the same argument can be used for spherical polygons, it proves the corresponding result.

The convexity of the area. In addition, Theorem $\mathrm{B}_{S}\left(\right.$ resp. $\left.\mathrm{B}_{H}\right)$ shows that the area is strictly concave over at least a subset of the space of convex polygons with given edge lengths. We call $A$ the function equal to the area of polygons.

Definition 4.6. Let $p$ be a convex polygon, with vertices $v_{1}, \cdots, v_{n}$. Let $\phi: \mathbb{R}^{3} \rightarrow$ $\mathbb{R}^{3}$ be defined by

$$
\phi(x)=\sum_{i=1}^{n}\left\langle x, v_{i}\right\rangle v_{i} .
$$

Suppose that the $v_{i}$ are not on a geodesic, let $C_{v}$ be the barycenter of the vertices of $p$; we set

$$
C_{S}(p):=\frac{\phi^{-1}\left(C_{v}\right)}{\left\|\phi^{-1}\left(C_{v}\right)\right\|} .
$$

Note that, since the $v_{i}$ are not on a geodesic, $\phi$ is a self-adjoint, positive definite operator, so it is invertible.

To understand the meaning of $C_{S}(p)$, we consider first the simplest case, when $p$ is a spherical triangle, with vertices $v_{1}, v_{2}$ and $v_{3}$, which we suppose are not on a geodesic. Then $C_{S}(p)$ is the barycenter of the dual polygon $p^{*}$. Indeed, let $p^{*}=\left(v_{1}^{*}, v_{2}^{*}, v_{3}^{*}\right)$; then $\left(v_{1}^{*}, v_{2}^{*}, v_{3}^{*}\right)$ is the basis of $\mathbb{R}^{3}$ which is dual to the basis $\left(v_{1}, v_{2}, v_{3}\right)$. By definition, $C_{S}(p)$ is defined by the equation

$$
\frac{\sum_{i=1}^{3}\left\langle v_{i}, C_{S}(p)\right\rangle v_{i}}{\left\|\sum_{i=1}^{3}\left\langle v_{i}, C_{S}(p)\right\rangle v_{i}\right\|}=C_{v}(p) .
$$

Taking the scalar product with $v_{j}^{*}$, we obtain that $C_{S}(p)$ is characterized by the existence of a $\lambda>0$ such that, for all $j \in\{1,2,3\}$,

$$
\left\langle C_{S}(p), v_{j}\right\rangle=\lambda\left\langle C_{v}(p), v_{j}^{*}\right\rangle .
$$

The action of the duality on this equation shows that $C_{S}(p)$ is the barycenter of the polygon $p^{*}=\left(v_{1}^{*}, v_{2}^{*}, v_{3}^{*}\right)$.

This example can be extended by taking a triangle with integer "weights", i.e. we consider a polygon with $k_{1}+k_{2}+k_{3}$ vertices, $k_{1}$ of them being "collapsed" on a point $v_{1}, k_{2}$ on $v_{2}$, and $k_{3}$ on $v_{3}$. The argument given above carries over to this case and, again in this case, the point $C_{S}(p)$ which is obtained is the barycenter of the triangle dual to $\left(v_{1}, v_{2}, v_{3}\right)$; in other terms, the weights $k_{1}, k_{2}, k_{3}$ have no influence. This illustrates an interesting "stability" property of $C_{S}(p)$.

The concavity of the area. Theorem $\mathrm{C}$ is related to the fact that, over at least part of $\mathcal{P}_{H}^{c}(l)\left(\right.$ resp. $\left.\mathcal{P}_{S}^{c}(l)\right)$, the area is strictly concave.

Lemma 4.7. Let $p$ be a convex polygon in $S^{2}$ (resp. $H^{2}$ ) with edge lengths given by $l=\left(l_{1}, \cdots, l_{n}\right)$. Suppose that $C_{S}(p)$ is contained in the interior of $p$. Then the restriction of $A$ to $\mathcal{P}_{S}^{c}(l)$ is strictly concave at $p$ for the metric induced by the immersion of $\mathcal{P}_{S}(l)$ in $[0, \pi]^{n}$ defined by sending a polygon to its angles.

Proof. First note that $A$ has an extension as an affine function over $[0, \pi]^{n}$ since, by the Gauss-Bonnet formula, $A=2 \pi-\sum_{i=1}^{n}\left(\pi-\alpha_{i}\right)$ for spherical polygons, while $A=-2 \pi+\sum_{i=1}^{n}\left(\pi-\alpha_{i}\right)$ for hyperbolic polygons. We still call this function $A$, 
then $d A=(1, \cdots, 1)$ for spherical polygons, while $d A=-(1, \cdots, 1)$ for hyperbolic polygons.

Let Hess $(A)$ be the Hessian of $A$ over $\Phi_{l}\left(\mathcal{P}_{S}(l)\right)$. Let $U$ be a first-order isometric deformation of $p$, then

$$
\operatorname{Hess}(A)(U, U)=d A\left(I I\left(d \Phi_{l}(U), d \Phi_{l}(U)\right)\right)=\left\langle I I\left(d \Phi_{l}(U), d \Phi_{l}(U)\right), I\right\rangle,
$$

where $I I$ is the second fundamental form of $\Phi_{l}\left(\mathcal{P}_{S}^{c}(l)\right)$ and $I$ is the vector $(1, \cdots, 1)$. Since $I I\left(d \Phi_{l}(U), d \Phi_{l}(U)\right)$ is orthogonal to $\Phi_{l}\left(\mathcal{P}_{S}^{c}(l)\right)$,

$$
\operatorname{Hess}(A)(U, U)=\left\langle I I\left(d \Phi_{l}(U), d \Phi_{l}(U)\right), I_{N}\right\rangle,
$$

where $I_{N}$ is the orthogonal projection of $I$ on the normal space of $\Phi_{l}\left(\mathcal{P}_{S}^{c}(l)\right)$ at $\Phi_{l}(p)$. By definition of $\Psi_{p}, I_{N}=\Psi_{p}(w)$, where $w \in \mathbb{R}^{3}$ is characterized by the fact that $\Psi_{p}(w)-I \in T_{\Phi_{l}(p)} \Phi_{l}\left(\mathcal{P}_{S}^{c}(l)\right)$, which translates as

$$
\sum_{i=1}^{n}\left(\left\langle w, v_{i}\right\rangle-1\right) v_{i}=0
$$

so that

$$
\sum_{i=1}^{n}\left\langle w, v_{i}\right\rangle v_{i}=\sum_{i=1}^{n} v_{i}
$$

This shows that $w=\lambda C_{S}(p)$, for some $\lambda>0$.

Applying Remark 3.5 then leads to

$$
\operatorname{Hess}(A)(U, U)=-\lambda\left\langle b(U), C_{S}(p)\right\rangle,
$$

so that the Hessian of $A$ is negative definite at $p$.

The proof in the spherical case is almost the same, with some differences in the signs, and we leave it to the interested reader.

Although the proof given here only works when $C_{S}(p)$ is contained in the interior of $p$, it is not obvious whether the result is valid for all convex polygons; the existence of a unique critical point of the area over $\mathcal{P}_{S}^{c}(l)$, which is true in all cases, suggests that it might be the case.

\section{INFINITESIMAL RIGIDITY OF EUCLIDEAN POLYHEDRA}

Infinitesimal rigidity of convex polyhedra. In this section we consider convex polyhedra in $\mathbb{R}^{3}$. We define a convex polyhedron as the boundary of a compact subset of $\mathbb{R}^{3}$ which is the intersection of a finite number of half-spaces. Given a polyhedron $P$, which is the boundary of a compact subset $\Omega \subset \mathbb{R}^{3}$, where $\Omega$ is the intersection of distinct closed half-spaces $H_{1}, \cdots, H_{n}$ (each of which intersect the interior of $\Omega$ ), a vertex of $P$ is a point of $P$ which is contained in at least 3 of the boundaries of the $H_{i}$. An edge of $P$ is a maximal connected subset of $P$ which is contained in the boundaries of two of the $H_{i}$ but containing no vertex. A face of $P$ is a connected component of the complement of the vertices and the edges.

The geometry of Euclidean polyhedra has interested geometers for quite a long time Euc02. A nice result of Legendre LegII 3 and Cauchy Cau13] states that convex polyhedra are rigid: If two convex polyhedra have the same combinatorics and the same induced metric on their faces, they are congruent. A related result, first proved by Dehn Deh16] (which is also a consequence of the ideas of Legendre and Cauchy) is that convex polyhedra are infinitesimally rigid: Any first-order

\footnotetext{
${ }^{3}$ The contribution of Legendre was recently pointed out by I. Sabitov Sab04].
} 
deformation of a polyhedron $P$ which acts as a Killing field on each of its faces is the restriction to $P$ of a global Killing field.

From a modern viewpoint, one of the main motivations to study the infinitesimal rigidity of convex polyhedra is that it is the key point in the proof of the following nice theorem.

Theorem 5.1 (Aleksandrov Ale58]). The induced metric on any convex polyhedron in $\mathbb{R}^{3}$ is flat, with conical singularities where the total angle is less than $2 \pi$. Conversely, any flat metric on $S^{2}$ with conical singularities where the total angle is less than $2 \pi$ is induced on a unique (up to isometries) convex polyhedron in $\mathbb{R}^{3}$.

This theorem is beyond the scope of this paper, but we can give a very broad outline of a possible proof. One could consider the natural map sending a convex polyhedron to its induced metrics; it is a map between two spaces of the same dimension, and the infinitesimal rigidity statement means that its differential is everywhere an isomorphism. This, along with other arguments, can then be used to show that it is a local homeomorphism.

We will show here that Theorem 1.4 is a consequence of Theorem $\mathrm{B}_{S}$, thus providing a new proof which is rather natural. It will also serve as an introduction for the rigidity result of the next section, since the proof given there is related but more complicated. The rigidity proof given here is close to a proof given by Milka Mil65, and is also related to a proof of the Cauchy-Legendre rigidity result discovered by Pogorelov Pog56. We describe it here for completeness, and as an introduction to the considerations of the next section.

Closed convex polyhedra. Let $P$ be a closed, convex Euclidean polyhedron. Choose a point $p_{0}$ in the interior of $P$. Let $u_{0}$ be the function defined on $\mathbb{R}^{3}$ by $u_{0}(p)=d\left(p_{0}, p\right)^{2} / 2$. We consider a first-order deformation of $P$, i.e., a vector field $v$ on $P$ which acts as a Killing field on each of its faces; we will show that $v$ is trivial, i.e., it acts as a Killing field on $P$ as a whole.

Let $e$ be an edge of $P$. The first-order deformation $v$ of $P$ determines a first-order variation $\dot{\theta}_{e}$ of the dihedral angle $\theta_{e}$ of $P$ at $e . v$ also determines a function, which we call $\dot{u}_{0}$, on $P$, defined as the first-order variation of the restriction of $u_{0}$ to $P$. By definition of $u_{0}$, we have

$$
\forall p \in P, \dot{u}_{0}(p)=\left\langle v(p), p_{0} p\right\rangle .
$$

We define a 1-form $W$ on the 1-skeleton of $P$ (the union of its edges) as follows: For each vector $u$ tangent to an edge $e, W(u)=\dot{\theta}_{e} d \dot{u}_{0}(u)$.

Consider a parametrization of $e$ at constant speed, say $p(t)=a t+b$, with $a, b \in \mathbb{R}^{3}$. Then, using (5.1),

$$
\dot{u}_{0}(p(t))^{\prime}=d \dot{u}_{0}\left(p^{\prime}(t)\right)=\left\langle d v\left(p^{\prime}(t)\right), p_{0} p(t)\right\rangle+\left\langle v(p(t)), p^{\prime}(t)\right\rangle .
$$

But $p^{\prime}(t)=a$ is independent of $t$, and $d v\left(p^{\prime}(t)\right)$ does not depend on $t$ either since $v$ acts on $e$ as a Killing field. So

$$
\frac{d}{d t} \dot{u}_{0}(p(t))^{\prime}=2\left\langle d v\left(p^{\prime}(t)\right), p^{\prime}(t)\right\rangle .
$$

But $v$ acts isometrically on $e$, so that $\left\langle d v\left(p^{\prime}(t)\right), p^{\prime}(t)\right\rangle=0$, and it follows that $d \dot{u}_{0}\left(p^{\prime}(t)\right)$ is constant over $e$, so $\dot{\theta}_{e} d \dot{u}_{0}\left(p^{\prime}(t)\right)$ is constant over $e$. 
Now consider an oriented edge $e$ of $P$, we call $e_{-}$and $e_{+}$its endpoints, and we let $W_{e}$ be the number $W\left(p^{\prime}(t)=\dot{\theta}_{e} d \dot{u}_{0}\left(p^{\prime}(t)\right)\right.$, where $p(t)$ is a parametrization of $e$ at speed 1 , respecting the orientation. Then we have

$$
\sum_{v} \sum_{e_{-}=v} W_{e}=0
$$

because each non-oriented edge contributes twice, with opposite signs.

Let $x$ be one of the vertices of $P$. Let $e_{1}, \cdots, e_{n-1}, e_{n}=e_{0}$ be the oriented edges of $P$ such that $e_{i,-}=x$, with their natural cyclic order, and let $v_{1}, \cdots, v_{n}=v_{0}$ be unit vectors with $v_{i}$ in the direction of $e_{i}$, oriented from $x$ towards the other vertex of $e_{i}$. Then $v_{1}, \cdots, v_{n}$ are the vertices of a spherical polygon (called the link of $P$ at $x$ ) which is convex since $P$ is convex.

For each $i \in\{1, \cdots, n\}$, the first-order deformation $v$ of $P$ determines a firstorder deformation $v^{\prime}$ of the spherical polygon $\left(v_{1}, \cdots, v_{n}\right)$, given at each vertex $v_{i}$ by the vector $\dot{v}_{i}=d v\left(v_{i}\right)$; note that $d v\left(v_{i}\right)$ is orthogonal to $v_{i}$ because $v$ is an isometric deformation. Since $v$ is isometric, it does not change (at first order) the interior angles of the faces of $P$; since the interior angles of the faces of $P$ at $x$ are the lengths of the edges of $\left(v_{1}, \cdots, v_{n}\right)$, the first-order deformation $v^{\prime}$ is isometric.

The angles $\alpha_{1}, \cdots, \alpha_{n}$ of the polygon $\left(v_{1}, \cdots, v_{n}\right)$ are equal to the dihedral angles $\theta\left(e_{1}\right), \cdots, \theta\left(e_{n}\right)$ of $P$ at the edges $e_{1}, \cdots, e_{n}$. Therefore,

$$
\begin{aligned}
\sum_{i=1}^{n} W_{e_{i}} & =\sum_{i=1}^{n}\left(\left\langle d v\left(v_{i}\right), p_{0} x\right\rangle+\left\langle v(x), v_{i}\right\rangle\right) \dot{\theta}_{i} \\
& =\sum_{i=1}^{n}\left(\left\langle\dot{v}_{i}, p_{0} x\right\rangle+\left\langle v(x), v_{i}\right\rangle\right) \dot{\alpha}_{i} \\
& =\left\langle\sum_{i=1}^{n} \dot{\alpha}_{i} \dot{v}_{i}, p_{0} x\right\rangle+\left\langle v(x), \sum_{i=1}^{n} \dot{\alpha}_{i} v_{i}\right\rangle .
\end{aligned}
$$

Since $p_{0}$ is in $P$ and $P$ is convex, $-p_{0} x$ is in the positive cone over the interior of $\left(v_{1}, \cdots, v_{n}\right)$, so that the first sum is non-positive by Theorem $\mathrm{B}_{S}$, and it vanishes if and only if the deformation $v^{\prime}$ is trivial. The second sum is zero by Theorem $\mathrm{A}_{S}$. So, by (5.2), the first sum is 0 for each vertex $x$ of $P$, and none of the angles of $P$ varies in the first-order deformation $v$, so that $v$ is a trivial deformation.

\section{Convex polyhedral surfaces in the Minkowski space}

This section contains an extension of Theorem 1.4 to a rigidity statement for convex, equivariant surfaces in the Minkowski space, for which the techniques used here are particularly well adapted since there is a distinguished point. Partial results on this rigidity question where obtained by I. Iskhakov [Isk00], by different methods. We first define the polyhedral surfaces that are considered. Then we basically follow the path taken above in the Euclidean case, adapting the proof from the sphere to the de Sitter plane. Along the way we recall some elementary facts of de Sitter geometry, proved here for completeness.

Equivariant embeddings of surfaces. Let $S$ be a closed surface, which will be of genus at least 2 in all this section. We first define equivariant polyhedral embeddings of $S$. More general definitions could of course be given, but we stick to what is really necessary in our context. 
Definition 6.1. An equivariant space-like polyhedral embedding of $S$ in $\mathbb{R}_{1}^{3}$ is a couple $(\phi, \rho)$, where:

- $\phi$ is a space-like polyhedral embedding of the universal cover $\tilde{S}$ of $S$ in $\mathbb{R}_{1}^{3}$, i.e.:

$-\phi$ is continous.

- There exists a cellular decomposition of $S$ as the union of a finite number of cells, each the image by a diffeomorphism of the interior of a convex polygon in $\mathbb{R}^{2}$, such that the image by $\phi$ of each cell of the lifted cellular decomposition of $\tilde{S}$ is the interior of a convex polygon is some space-like plane in $\mathbb{R}_{1}^{3}$.

- For each space-like plane $H$ in $\mathbb{R}_{1}^{3}$, the orthogonal projection of $\phi(\tilde{S})$ on $H$ is one-to-one.

- $\rho$ is a morphism from $\pi_{1}(S)$ to the isometry group of $\mathbb{R}_{1}^{3}$.

- For each $x \in \tilde{S}$ and each $\gamma \in \pi_{1}(S), \phi(\gamma x)=\rho(\gamma) \phi(x)$.

We will say that $(\phi, \rho)$ is Fuchsian if the image of $\rho$ is contained in the identity component of the subgroup of isometries fixing the origin, i.e. $\rho\left(\pi_{1}(S)\right) \subset S O_{0}(2,1)$.

A direct consequence of the definition of an equivariant embedding of $S$ in $\mathbb{R}_{1}^{3}$ is that the metric induced on $\tilde{S}$ by $\phi$ is invariant under the action of $\pi_{1} S$, so that an equivariant embedding of $S$ induces a metric on $S$.

Consider an equivariant embedding $(\phi, \rho)$ of $S$ in $\mathbb{R}_{1}^{3}$. There is a natural notion of first-order deformation of $(\phi, \rho)$; it corresponds to the deformations of $\phi$ among the equivariant embeddings of $S$. Restricting our attention to the deformations among Fuchsian equivariant embeddings, such a deformation can be described as a couple $(\dot{\phi}, \dot{\rho})$, where:

- $\dot{\phi}$ is a vector field defined over $\phi(\tilde{S})$.

- $\dot{\rho}: \pi_{1} S \rightarrow T S O(2,1)$ is a map, such that

$$
\forall \gamma \in \pi_{1} S, \dot{\rho}(\gamma) \in T_{\rho(\gamma)} S O_{0}(2,1),
$$

and, for all $\gamma, \gamma^{\prime} \in \pi_{1} S$, if we consider $\rho(\gamma)$ and $\rho\left(\gamma^{\prime}\right)$ as acting on $S O(2,1)$ and $\dot{\rho}$ as sending elements of $\pi_{1} S$ to vector fields on $S O(2,1)$, we have

$$
\dot{\rho}\left(\gamma \gamma^{\prime}\right)=\dot{\rho}(\gamma)\left(\rho\left(\gamma^{\prime}\right)\right)+\rho(\gamma)_{*} \dot{\rho}\left(\gamma^{\prime}\right) .
$$

- If we identify the elements of $s o(2,1)$ with the Killing fields on $\mathbb{R}_{1}^{3}$ which vanish at 0 , then

$$
\forall x \in \tilde{S}, \forall \gamma \in \pi_{1} S, \dot{\phi}(\gamma x)=\rho(\gamma)_{*} \dot{\phi}(x)+\dot{\rho}(\gamma) \phi(x) .
$$

Fuchsian polyhedral surfaces. The main result of this section is the following infinitesimal rigidity statement. There is an analogous statement for smooth surfaces in LS00.

Theorem 6.2. Let $(\phi, \rho)$ be a Fuchsian, convex equivariant embedding of a surface $S$ (of genus at least 2 ) in $\mathbb{R}_{1}^{3}$. Let $(\dot{\phi}, \dot{\rho})$ be a first-order deformation of $(\phi, \rho)$ among Fuchsian equivariant embeddings. If the first-order variation of the metric induced on $S$ by $(\phi, \rho)$ vanishes, then $(\dot{\phi}, \dot{\rho})$ is trivial, i.e. $\dot{\phi}$ is the restriction to $\phi(\tilde{S})$ of a Killing field of $\mathbb{R}_{1}^{3}$ which vanishes at 0 , and $\dot{\rho}=0$. 
The proof comes after some preliminaries, basically following the proof of Theorem 1.4 and checking that the various parts of the proof carry over from the Euclidean to the Minkowski 3-dimensional space, and from the sphere to the de Sitter plane.

As in the Euclidean case, the main motivation for this theorem is that it should be a key point in the proof of a statement describing the metrics induced on fuchsian equivariant polyhedra in $\mathbb{R}_{1}^{3}$, as in the Aleksandrov theorem quoted above for the Euclidean space. Namely, one would like to answer the following question:

Let $S$ be a surface of genus at least 2 , and let $g$ be a flat metric on $S$ with conical singularities, with total angle larger than $2 \pi$ at each singular point. Is there a unique (up to global isometries) convex Fuchsian polyhedral embedding of $S$ in $\mathbb{R}_{1}^{3}$ such that the induced metric is $g$ ?

We do not answer this question here since it would demand some considerations leading us too far from Theorems $\mathrm{A}_{S}$ and $\mathrm{B}_{S}$, the main theme of this text.

Distances and angles in the de Sitter plane. As mentioned in the introduction, the de Sitter plane, denoted by $S_{1}^{2}$, is the quadric of equation $\langle x, x\rangle=1$ in $\mathbb{R}_{1}^{3}$, with the induced metric. Details can be found e.g. in Cox43, Cox57, O'N83.

Let $x, x^{\prime}$ be two points in $S_{1}^{2}$. By analogy with the distance in the sphere $S^{2} \subset \mathbb{R}^{3}$, we define the "distance" between $x$ and $x^{\prime}$ as the number $d\left(x, x^{\prime}\right) \in \mathbb{C} / 2 \pi \mathbb{Z}$ such that

$$
\left\langle x, x^{\prime}\right\rangle=\cos \left(d\left(x, x^{\prime}\right)\right) .
$$

This leaves an indetermination concerning the sign of $d\left(x, x^{\prime}\right)$, which is removed by the following explicit description:

- $d\left(x, x^{\prime}\right) \in(0, \pi)$ when $x$ and $x^{\prime}$ are distinct, non-antipodal points on a space-like geodesic.

- $d\left(x, x^{\prime}\right)=0$ when $x$ and $x^{\prime}$ are on a light-like geodesic.

- $d\left(x, x^{\prime}\right) \in i \mathbb{R}_{+}$when $x$ and $x^{\prime}$ are on a time-like geodesic.

- $d\left(x, x^{\prime}\right) \in \pi-i \mathbb{R}_{+}$when $x$ and $x^{\prime}$ are in two different connected components of the intersection of $S_{1}^{2}$ with a time-like plane or light-like plane containing 0 (each connected component of the intersection is then a time-like or lightlike geodesic).

This definition differs from the one used in [Sch98] by a factor $i$, basically because here we stick as close as possible to spherical geometry, while the emphasis in Sch98. was on hyperbolic geometry. The definition of [Sch98] would be obtained if we had taken the cosh of $d\left(x, x^{\prime}\right)$ instead of the cosine in the definition above. The proof below would then have less factors " $i$ ", but it would be based on the hyperbolic rather than the spherical trigonometric formulas, thus blurring the analogy with the proof of Theorem C.

There is a related notion of angles in $S_{1}^{2}$. Let $x \in S_{1}^{2}$ and let $u, v \in T_{x} S_{1}^{2}$ be such that $\langle u, u\rangle \neq 0$ and $\langle v, v\rangle \neq 0$; the angle between them is a number $\theta \in \mathbb{C} / 2 \pi \mathbb{Z}$ such that

$$
\langle u, v\rangle^{2}=\cos ^{2}(\theta)\langle u, u\rangle\langle v, v\rangle .
$$

Note that $\theta$ is defined up to sign and up to the transformation $\theta \mapsto \pi-\theta$, which is quite normal since it depends on the direction of $u$ and $v$. This ambiguity is 
removed by the following explicit description, in which we suppose that $(u, v)$ is a positively oriented basis of $T_{x} S_{1}^{2}$ :

- $\theta \in i \mathbb{R}_{+}$if $u$ and $v$ are both future-oriented time-like vector (or if they are both past-oriented time-like vectors).

- $\theta \in \pi-i \mathbb{R}_{+}$if $u$ and $v$ are time-like vectors, but $u$ is future-oriented and $v$ is past-oriented.

- $\theta \in i \mathbb{R}_{+}$if $u$ and $v$ are both space-like vectors, and are in the same connected component of the set of space-like vectors at $x$.

- $\theta \in \pi-i \mathbb{R}_{+}$if $u$ and $v$ are space-like vectors in different connected components of the set of space-like vectors at $x$.

- $\theta \in \pi / 2+i \mathbb{R}$ if $u$ is space-like and $v$ is time-like, or conversely.

Triangle formulas in the de Sitter plane. With those definitions, equation (3.1) and the sine formula hold in $S_{1}^{2}$ exactly as in the sphere.

Proposition 6.3. Let $(A, B, C)$ be a triangle in the de Sitter plane, with edge lengths $a, b, c$ and angles $\alpha, \beta, \gamma$. Suppose that $a, b, c \neq 0$. Then

$$
\cos (a)=\cos (b) \cos (c)+\cos (\alpha) \sin (b) \sin (c) .
$$

Moreover, if $a, b, c \neq 0$, then

$$
\frac{\sin (\alpha)}{\sin (a)}=\frac{\sin (\beta)}{\sin (b)}=\frac{\sin (\gamma)}{\sin (c)}
$$

Proof. Recall the definition of the cross-product $X \otimes Y$, in coordinates, used in the Minkowski space

$$
\left(x_{1}, x_{2}, x_{3}\right) \otimes\left(y_{1}, y_{2}, y_{3}\right)=\left(x_{2} y_{3}-x_{3} y_{2}, x_{3} y_{1}-x_{1} y_{3},-x_{1} y_{2}+x_{2} y_{1}\right) .
$$

Note that this definition is natural insofar as it can be obtained like the cross product in Euclidean 3 -space, by associating 1 -forms to vectors, taking the wedge product, and associating a vector to the resulting 2 -form. This shows that the definition given using coordinates is independent of the orthonormal basis of $\mathbb{R}_{1}^{3}$ which has been used.

It follows from this definition that, given 3 vectors $X, Y$ and $Z$ in $\mathbb{R}^{3}$, the number $\langle X, Y \otimes Z\rangle$ is the same whether the cross product and the scalar product are considered for the Euclidean or the Minkowski space structure (there are two sign differences but they cancel out). Therefore, it remains true in the Minkowski space that

$$
\langle X, Y \otimes Z\rangle=\langle Y, Z \otimes X\rangle=\langle Z, X \otimes Y\rangle .
$$

The same argument also shows that $\langle X, X \otimes Y\rangle=\langle Y, X \otimes Y\rangle=0$ in the Minkowski space, so that $X \otimes Y$ is orthogonal (for the Minkowski scalar product) to $X$ and to $Y$.

It is also easy to check, by taking a "good" orthonormal basis of $\mathbb{R}_{1}^{3}$, that, if $X$ is a space-like unit vector orthogonal to $Y$ and $Z$, then

$$
\langle X \otimes Y, X \otimes Z\rangle=\langle Y, Z\rangle \text {. }
$$

Moreover, let $X, Y \in S_{1}^{2}$ be two non-collinear vectors of $\mathbb{R}_{1}^{3}$, then a direct computation shows that

$$
\|X \otimes Y\|^{2}=-\|X\|^{2}\|Y\|^{2}+\langle X, Y\rangle^{2},
$$


from which a careful analysis of different cases (depending on whether $X$ and $Y$ are contained in a space-like or time-like geodesic) shows that, if $N$ is a vector orthogonal to $X$ and $Y$ with $\|N\|^{2}= \pm 1$, then

$$
X \otimes Y= \pm \sin (d(X, Y)) N .
$$

Now let $A, B, C \subset S_{1}^{2}$ be the 3 vertices of a de Sitter triangle. Then $B$ and $C$ can be decomposed as $B=B_{\|} A+B_{\perp}$ and $C=C_{\|} A+C_{\perp}$, where $B_{\|}, C_{\|} \in \mathbb{R}$ and $B_{\perp}, C_{\perp} \in \mathbb{R}_{1}^{3}$ are vectors orthogonal to $A$. Then

$$
\begin{array}{r}
\langle A \otimes B, A \otimes C\rangle=\left\langle A \otimes\left(B_{\|} A+B_{\perp}\right), A \otimes\left(C_{\|} A+C_{\perp}\right)\right\rangle=\left\langle A \otimes B_{\perp}, A \otimes C_{\perp}\right\rangle \\
=\left\langle B_{\perp}, C_{\perp}\right\rangle=\langle B, C\rangle-B_{\|} C_{\|}\langle A, A\rangle=\langle B, C\rangle-\langle A, B\rangle\langle A, C\rangle .
\end{array}
$$

But $A \otimes B=\sin (c) N_{C}$ and $A \otimes C=\sin (b) N_{B}$, where $N_{C}$ and $N_{B}$ are unit vectors orthogonal to the plane containing $0, A, B$ and $0, A, C$, respectively. By definition, $\left\langle N_{C}, N_{B}\right\rangle=\cos (\alpha)$, and we find that

$$
\begin{aligned}
\cos (\alpha) \sin (b) \sin (c) & =\langle A \otimes B, A \otimes C\rangle=\langle B, C\rangle-\langle A, B\rangle\langle A, C\rangle \\
& =\cos (a)-\cos (b) \cos (c),
\end{aligned}
$$

which proves the first equation.

To prove the sine formula, note that $N_{B}$ and $N_{C}$ are both orthogonal to $A$, so that $(A \otimes B) \otimes(A \otimes C)$ is collinear to $A$. It follows that

$$
\langle(A \otimes B) \otimes(A \otimes C), A\rangle=\sin (c) \sin (b) \sin (\alpha) .
$$

But, using the same decomposition of $B$ and $C$ as above, we have

$$
\begin{aligned}
(A \otimes B) \otimes(A \otimes C) & =\left(A \otimes B_{\perp}\right) \otimes\left(A \otimes C_{\perp}\right)=B_{\perp} \otimes C_{\perp} \\
& =B \otimes C-B_{\|}\left(A \otimes C_{\perp}\right)-C_{\|}\left(A \otimes B_{\perp}\right) ;
\end{aligned}
$$

taking the scalar product with $A$ yields

$$
\langle(A \otimes B) \otimes(A \otimes C), A\rangle=\langle B \otimes C, A\rangle,
$$

so that

$$
\langle B \otimes C, A\rangle=\sin (b) \sin (c) \sin (\alpha) .
$$

It follows that the quantity $\sin (b) \sin (c) \sin (\alpha)$ is invariant under a cyclic permutation on $(a, b, c)$ and $(\alpha, \beta, \gamma)$, and the second equation in the proposition follows.

Polygons in the de Sitter plane. The analog of Theorem $\mathrm{A}_{S}$ and Theorem $\mathrm{A}_{H}$ also holds for de Sitter polygons with non-degenerate edges (when one of the edges is degenerate, the notion of angle is not well-defined, so that the statements would not make sense).

Theorem $\mathbf{A}_{d S}$. Let $p=\left(v_{1}, \cdots, v_{n}\right)$ be a de Sitter polygon with non-degenerate edges. Let $\alpha_{1}, \cdots, \alpha_{n}$ be its angles, and let $\dot{\alpha}_{1}, \cdots, \dot{\alpha}_{n} \in \mathbb{R}$ be a first-order variation of its angles induced by an isometric first-order deformation of $p$. Then

$$
\sum_{i=1}^{n} \dot{\alpha}_{i} v_{i}=0,
$$

where the $v_{i}$ are considered as points in $S_{1}^{2} \subset \mathbb{R}_{1}^{3}$.

Conversely, if this equation is satisfied by an $n$-uple $\left(\dot{\alpha}_{1}, \cdots, \dot{\alpha}_{n}\right)$ and moreover the $v_{i}$ are not all on a de Sitter geodesic-or on the intersection of $S_{1}^{2}$ with a 
Minkowski plane containing the origin, then there exists an isometric first-order deformation of $p$ such that the $\dot{\alpha}_{i}$ are the associated first-order variations of the $\alpha_{i}$.

The proof follows exactly the proof of Theorem $\mathrm{A}_{H}$.

Again as in the sphere, Theorem $\mathrm{A}_{d S}$ leads to the definition of a quadratic invariant $b$ defined on the first-order isometric deformations of a de Sitter polygon (with non-degenerate edges). $b$ is defined as

$$
b(U)=\sum_{i=1}^{n} d \alpha_{i}(U) d v_{i}(U),
$$

As in the spherical and the hyperbolic case, if two first-order infinitesimal deformations $U$ and $U^{\prime}$ differ by a trivial deformation, then $b(U)=b\left(U^{\prime}\right)$. However, the definition of the angles which we have used means that $b(U)$ is imaginary.

The positivity of $b$. In the same way, the "positivity" property of $b$ which we found in the spherical case still holds for the de Sitter polygons which are duals of convex hyperbolic polygons.

Theorem $\mathbf{B}_{d S}$. Let $p$ be a strictly convex polygon in $S_{1}^{2}$, which is dual to a convex hyperbolic polygon $p^{*}$, and let $U$ be a non-trivial infinitesimal first-order deformation of $p$. Then $i b(U) \in \mathbb{R}_{+}^{*}$ int $\left(p^{*}\right)$, i.e. $i b(U)$ is contained in the positive cone over the interior of $p^{*}$.

The proof follows the proof of Theorem $\mathrm{B}_{S}$, but some additional details are necessary. Using Proposition 6.3, the computations done in Section 3 carry over to the de Sitter case. In particular, the scalar product of $b(U)$ with $v_{1}$ remains diagonal in the basis $\left(U_{2}, \cdots, U_{n-2}\right)$. Equation (3.6) can be rewritten in a way which is more convenient for us, as

$$
\left\langle b\left(U_{i}\right), v_{1}\right\rangle=\frac{\sin ^{2}\left(d_{1, i+1}\right) \sin \left(\alpha_{1}^{\prime}\right)}{\sin \left(d_{0, i+1}\right) \sin \left(d_{i, i+1}\right) \sin \left(\alpha_{0}^{\prime}\right) \sin \left(\alpha_{i}^{\prime}\right)}\left(d d_{1, i+1}\left(U_{i}\right)\right)^{2} .
$$

This follows from (3.6) because, in Lemma 3.3, the definition of $V$ is that, under this first-order variation, the distance $d_{1, i+1}$ between $v_{1}$ and $v_{i+1}$ varies at speed 1 ; this also uses the fact that $b$ is a quadratic form. Recall that, in this equation $\alpha_{0}^{\prime}, \alpha_{1}^{\prime}$ and $\alpha_{i}^{\prime}$ are not the angles of the polygon $p$, but rather the angles of the quadrilateral $\left(v_{0}, v_{1}, v_{i}, v_{i+1}\right)$ at the vertices $v_{0}, v_{1}$ and $v_{i}$.

Another way to write this formula is

$$
\left\langle b\left(U_{i}\right), v_{1}\right\rangle=\frac{\sin \left(\alpha_{1}^{\prime}\right)}{\sin \left(d_{0, i+1}\right) \sin \left(d_{i, i+1}\right) \sin \left(\alpha_{0}^{\prime}\right) \sin \left(\alpha_{i}^{\prime}\right)}\left(d\left\langle v_{1}, v_{i+1}\right\rangle\left(U_{i}\right)\right)^{2} .
$$

Clearly, $\left(d\left\langle v_{1}, v_{i+1}\right\rangle\left(U_{i}\right)\right)^{2} \in \mathbb{R}_{+} ;$moreover, the edges of $p$ are space-like, $d_{i, i+1} \in$ $(0, \pi)$, so that $\sin \left(d_{i, i+1}\right) \in(0,1)$. However, to understand the signs of the various sines appearing in this equation, it is necessary to consider four different cases, depending on whether $d_{1, i}$ and $d_{0, i+1}$ are in $(0, \pi)$ or in $\pi-i \mathbb{R}_{+}^{*}$, and then the limit cases. The four main cases are shown, in the projective model of half of $S_{1}^{2}$, in Figure 3.

1st case: $d_{1, i} \in(0, \pi), d_{0, i+1} \in(0, \pi)$. In other terms, $v_{0}$ and $v_{i+1}$ are on a spacelike geodesic, and $v_{1}$ and $v_{i}$ are on a space-like geodesic. Then $\sin \left(d_{0, i+1}\right) \in(0,1)$. Moreover, the angles $\alpha_{0}^{\prime}, \alpha_{1}^{\prime}$ and $\alpha_{i}^{\prime}$ are all of the form either $i r$ or $\pi-i r$, for some 


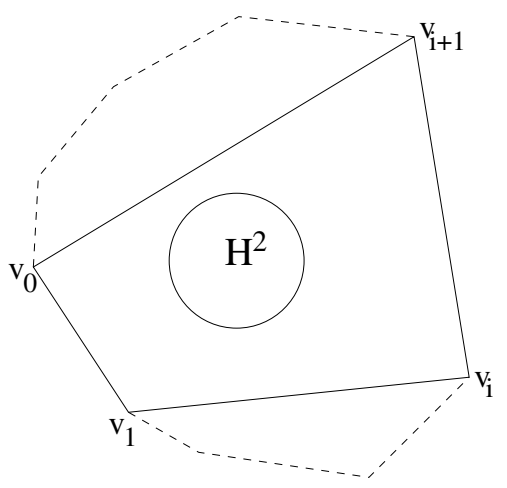

Case 1

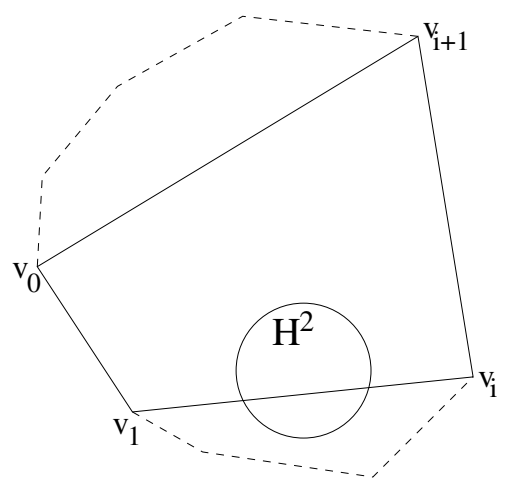

Case 3

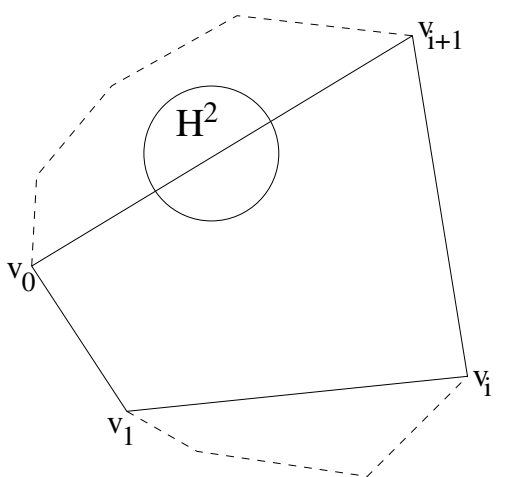

Case 2

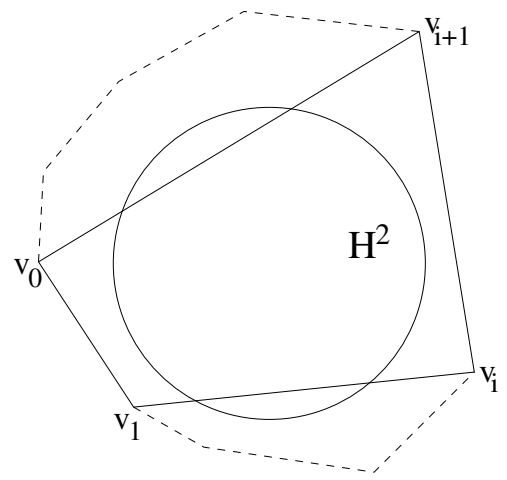

Case 4

Figure 3. Four possible cases

$r>0$, so that $\sin \left(\alpha_{0}^{\prime}\right), \sin \left(\alpha_{1}^{\prime}\right)$ and $\sin \left(\alpha_{i}^{\prime}\right)$ are in $i \mathbb{R}_{+}^{*}$. So equation (6.3) shows that $\left\langle b\left(U_{i}\right), v_{1}\right\rangle \in-i \mathbb{R}_{+}^{*}$.

2nd case: $d_{1, i} \in(0, \pi), d_{0, i+1} \in \pi-i \mathbb{R}_{+}^{*}$. This means that $v_{1}$ and $v_{i}$ are on a space-like geodesic, while $v_{0}$ and $v_{i+1}$ are on the intersection with $S_{1}^{2}$ of a time-like plane in $\mathbb{R}_{1}^{3}$ containing 0 (but on different connected components of this intersection). Now $\sin \left(d_{0, i+1}\right) \in i \mathbb{R}_{+}^{*} \cdot \alpha_{1}^{\prime}$ and $\alpha_{i}^{\prime}$ are of the form $\pi-i r$ for some $r>0$, so that $\sin \left(\alpha_{1}^{\prime}\right)$ and $\sin \left(\alpha_{i}^{\prime}\right)$ are in $i \mathbb{R}_{+}^{*}$, but $\alpha_{0}^{\prime}$ is of the form $\pi / 2+i r$, for some $r \in \mathbb{R}$, and it follows that $\sin \left(\alpha_{0}^{\prime}\right)=\cos (i r)=\cosh (r) \in[1, \infty)$. Therefore, (6.3) again shows that $\left\langle b\left(U_{i}\right), v_{1}\right\rangle \in-i \mathbb{R}_{+}^{*}$.

3rd case: $d_{1, i} \in \pi-i \mathbb{R}_{+}^{*}, d_{0, i+1} \in(0, \pi)$. Then $\sin \left(d_{0, i+1}\right) \in(0,1)$. Moreover, $\alpha_{0}^{\prime}$ is of the form $\pi-i r$, for some $r>0$, while $\alpha_{1}^{\prime}$ and $\alpha_{i}^{\prime}$ are of the form $\pi / 2+i r$, for some $r \in \mathbb{R}$. So $\sin \left(\alpha_{0}^{\prime}\right) \in i \mathbb{R}_{+}^{*}$, while $\sin \left(\alpha_{1}^{\prime}\right), \sin \left(\alpha_{i}^{\prime}\right) \in[1, \infty)$. By (6.3), $\left\langle b\left(U_{i}\right), v_{1}\right\rangle \in-i \mathbb{R}_{+}^{*}$ also in this case.

4th case: $d_{1, i} \in \pi-i \mathbb{R}_{+}^{*}, d_{0, i+1} \in \pi-i \mathbb{R}_{+}^{*}$. In this case, $\sin \left(d_{0, i+1}\right) \in i \mathbb{R}_{+}^{*}$, and $\alpha_{0}^{\prime}, \alpha_{1}^{\prime}$ and $\alpha_{i}^{\prime}$ are of the form $\pi / 2+i r$, for some $r \in \mathbb{R}$. Therefore, $\sin \left(\alpha_{0}^{\prime}\right), \sin \left(\alpha_{1}^{\prime}\right)$ and $\sin \left(\alpha_{i}^{\prime}\right)$ are in $[1, \infty)$, and, again, $\left\langle b\left(U_{i}\right), v_{1}\right\rangle \in-i \mathbb{R}_{+}^{*}$.

Last case: $v_{1}$ and $v_{i}$ are in the intersection with $S_{1}^{2}$ of a light-like plane in $S_{1}^{2}$ containing 0 , or the same holds for $v_{0}$ and $v_{i+1}$. The result then follows from an approximation of the quadrilateral $\left(v_{0}, v_{1}, v_{i}, v_{i+1}\right)$ by a sequence of 
quadrilaterals which are in one of the four cases detailed above, and a corresponding sequence of approximations of $U_{i}$.

Summing over $i \in\{2, \cdots, n-2\}$, we find that

$$
\left\langle i b(U), v_{1}\right\rangle=\sum_{i=2}^{n-2} i\left\langle b\left(U_{i}\right), v_{1}\right\rangle>0 .
$$

The same holds with $v_{1}$ replaced by any of the other vertices of $p$, and Theorem $\mathrm{B}_{d S}$ follows.

Proof of the rigidity theorem. The proof of Theorem 6.2 follows quite precisely the proof of Theorem C. We consider a Fuchsian, equivariant, convex polyhedral embedding $(\phi, \rho)$ of a surface $S$ of genus at least 2 in $\mathbb{R}_{1}^{3}$. Let $p_{0}=0 \subset \mathbb{R}_{1}^{3}$, i.e. $p_{0}$ is the point which is fixed by $\rho$. We call $u_{0}$ the function, defined on $\mathbb{R}_{1}^{3}$, as $u_{0}(x)=\langle x, x\rangle / 2$. Then, since 0 is fixed by the representation $\rho$, the restriction of $u_{0}$ to $\phi(\tilde{S})$ is invariant under the action of $\pi_{1} S$ by $\rho$, so that $u_{0}$ defines a function over $S$.

Let $(\dot{\phi}, \dot{\rho})$ be a first-order deformation of $(\phi, \rho)$ among Fuchsian embeddings. Let $e$ be an oriented edge of $\phi(\tilde{S})$, let $\theta_{e}$ be the corresponding dihedral angle, and let be $\dot{\theta}_{e}$ be the first-order variation of this dihedral angle under the first-order variation $(\dot{\phi}, \dot{\rho})$. We associate to $e$ the number $W_{e}$ defined as $W_{e}=\dot{\theta}_{e} d \dot{u}_{0}\left(p^{\prime}(t)\right)$, where $p(t)$ is a parametrization of $e$ at speed one. As in the Euclidean case - and for the same reasons $-W_{e}$ is independent of $t$. By construction, this quantity is invariant under the action of $\pi_{1} S$.

Again in this setting, $\sum_{v} \sum_{e_{-}=v} W_{e}=0$. On the other hand, let $x$ be a vertex of $\phi(\tilde{S})$. Let $e_{1}, \cdots, e_{n}$ be the oriented edges starting from $x$, in the cyclic order in which they appear. The link of $\phi(\tilde{S})$ at $x$ is a convex space-like polygon $p$, with vertices $v_{1}, \cdots, v_{n}$, corresponding to $e_{1}, \cdots, e_{n}$, which is the dual of a hyperbolic polygon. The first-order isometric deformation $(\dot{\phi}, \dot{\rho})$ induces a first-order deformation $U$ of $p$. Since $(\dot{\phi}, \dot{\rho})$ is isometric, it does not change (at first order) the interior angles of the faces of $\phi(\tilde{S})$ adjacent to $x$, so that the first-order deformation $U$ is isometric.

As in the Euclidean case, if $\dot{v}_{i}$ is the first-order displacement of $v_{i}$ under $U$ and if $\alpha_{i}$ is the angle of $p$ at $v_{i}$, then

$$
\begin{aligned}
\sum_{i=1}^{n} W_{e_{i}} & =\sum_{i=1}^{n}\left(\left\langle d \dot{\phi}\left(v_{i}\right), x\right\rangle+\left\langle\dot{\phi}(x), v_{i}\right\rangle\right) \dot{\theta}_{e_{i}} \\
& =\sum_{i=1}^{n}\left(\left\langle\dot{v}_{i}, x\right\rangle+\left\langle\dot{\phi}(x), v_{i}\right\rangle\right) \dot{\alpha}_{i} \\
& =\left\langle\sum_{i=1}^{n} \dot{\alpha}_{i} \dot{v}_{i}, x\right\rangle+\left\langle\dot{\phi}(x), \sum_{i=1}^{n} \dot{\alpha}_{i} v_{i}\right\rangle .
\end{aligned}
$$

The second term vanishes by Theorem $\mathrm{A}_{d S}$. By convexity of $\phi(\tilde{S}), x$ is in the interior of $p$, while, by Theorem $\mathrm{B}_{d S}, i b(U)$ is in the interior of the dual polygon $p^{*}$. It follows that $i \sum_{i=1}^{n} W_{e_{i}} \geq 0$, with equality if and only if the first-order deformation $U$ is trivial. The end of the proof is the same as in the Euclidean case.

Note that the rigidity argument given here for equivariant polyhedral embeddings in $\mathbb{R}_{1}^{3}$ could perhaps be used in other situations, for instance for closed, convex 
polyhedra in $\mathbb{R}_{1}^{3}$ which have faces which are not necessarily space-like. However the infinitesimal rigidity of convex polyhedra in $\mathbb{R}_{1}^{3}$ - even when some faces are not space-like - follows from the infinitesimal rigidity of convex polyhedra in the Euclidean space (see e.g. [Sch01]), so that having a direct Minkowski proof is not so important.

\section{ACKNOWLEDGEMENTS}

I would like to thank Philippe Eyssidieux, Vincent Guirardel, Sergiu Moroianu, and Boris Springborn for some helpful discussions and interesting comments related to the content of this paper. The text was also much improved thanks to many helpful comments from an anonymous referee.

\section{REFERENCES}

[Ale58] A. D. Alexandrow. Konvexe polyeder. Akademie-Verlag, Berlin, 1958. MR0092989 (19:1192c)

[Ale03] Victor Alexandrov. Flexible polyhedra in Minkowski 3-space. Manuscripta Math., 111(3):341-356, 2003. MR.1993499 (2004g:52035)

[Cau13] Augustin Louis Cauchy. Sur les polygones et polyèdres, second mémoire. Journal de l'Ecole Polytechnique, 19:87-98, 1813.

[Cox43] H. S. M. Coxeter. A geometrical background for de Sitter's world. Amer. Math. Monthly, 50:217-228, 1943. MR0007991 (4:226c)

[Cox57] H. S. M. Coxeter. Non-Euclidean geometry. Mathematical Expositions, no. 2. University of Toronto Press, Toronto, Ont., 1957. 3rd ed. MR0087965 (19:445e)

[Cox93] H. S. M. Coxeter. The real projective plane. Springer-Verlag, New York, third edition, 1993. With an appendix by George Beck, With 1 IBM-PC floppy disk (5.25 inch; DD). MR.1198272 (94b:51002b)

[Deh16] M. Dehn. Über den Starrheit konvexer Polyeder. Math. Ann., 77:466-473, 1916. MR 1511873

[Euc02] Euclid. Elements. Green Lion Press, Santa Fe, NM, 2002. All thirteen books complete in one volume, The Thomas L. Heath translation, Edited by Dana Densmore. MR1932864 (2003j:01044)

[Fil92] P. Filliman. Rigidity and the Alexandrov-Fenchel inequality. Monatsh. Math., 113(1):122, 1992. MR.1149057 (93c:52020)

[Glu75] Hermann Gluck. Almost all simply connected closed surfaces are rigid. Geometric topology (Proc. Conf., Park City, Utah, 1974), pp. 225-235, Lecture Notes Math., Vol. 438, Springer, Berlin, 1975. MR0400239 (53:4074)

[Isk00] I. Iskhakov. On hyperbolic surface tessellations and equivariant spacelike convex polyhedral surfaces in Minkowski space. Ph.D. thesis, Ohio State University, 2000.

[Kan90] Edgar Kann. Infinitesimal rigidity of almost-convex oriented polyhedra of arbitrary Euler characteristic. Pacific J. Math., 144(1):71-103, 1990. MR1056667(92a:52032)

[KM95] Michael Kapovich and John Millson. On the moduli space of polygons in the Euclidean plane. J. Differential Geom., 42(2):430-464, 1995. MR.1366551 (96k:58035)

[KM99] Michael Kapovich and John J. Millson. On the moduli space of a spherical polygonal linkage. Canad. Math. Bull., 42(3):307-320, 1999. MR1703691 (2000g:14020)

[LegII] A.-M. Legendre. Eléments de géométrie. Paris, 1793 (an II). Première édition, note XII, pp.321-334.

[LS00] François Labourie and Jean-Marc Schlenker. Surfaces convexes fuchsiennes dans les espaces lorentziens à courbure constante. Math. Annalen, 316:465-483, 2000. MR 1752780 (2001g:53127)

[Mil65] A. D. Milka. An analog of Blaschke's formula for polyhedra. Ukrain. Geometr. Sb. Vyp., 1:62-64, 1965. MR0215184(35:6027)

[O'N83] B. O'Neill. Semi-Riemannian Geometry. Academic Press, 1983. MR0719023 (85f:53002)

[Pog56] A. V. Pogorelov. A new proof of rigidity of convex polyhedra. Uspehi Mat. Nauk (N.S.), 11(5(71)):207-208, 1956. MR0083766 (18:758c) 
[RH93] Igor Rivin and Craig D. Hodgson. A characterization of compact convex polyhedra in hyperbolic 3-space. Invent. Math., 111:77-111, 1993. MR1193599 (93j:52015)

[Riv86] Igor Rivin. Thesis. Ph.D. thesis, Princeton University, 1986.

[Sab04] I. Kh. Sabitov. Around the proof of the Legendre-Cauchy lemma on convex polygons. Sibirsk. Mat. Zh., 45(4):892-919, 2004. Translated in Siberian Math. Journal 45:4 (2004), pp. 740-762. MR2091654(2005h:52002)

[Sch98] Jean-Marc Schlenker. Métriques sur les polyèdres hyperboliques convexes. J. Differential Geom., 48(2):323-405, 1998. MR1630178 (2000a:52018)

[Sch01] Jean-Marc Schlenker. Convex polyhedra in Lorentzian space-forms. Asian J. of Math., 5:327-364, 2001. MR 1868937 (2003f:53127)

[Sch03] Jean-Marc Schlenker. Des immersions isométriques de surfaces aux variétés hyperboliques à bord. In Séminaire de Théorie Spectrale et Géométrie, No. 21, Année 2002-2003, pages 165-216. Univ. Grenoble I, 2003. MR2052831 (2005c:53089)

[Sch04] Jean-Marc Schlenker. Small deformations of polygons. math.DG/0410058, v2, 2004.

[Sie02] A. Siegel. A Dido problem as modernized by Fejes Tóth. Discrete Comput. Geom., 27(2):227-238, 2002. MR:1880939 (2002m:52009)

[Ste42] J. Steiner. Sur le maximum et le minimum des figures dans le plan, sur la sphère, et dans l'espace en général. J. Reine Angew. Math., 24:93-152, 190-250, 1842.

[Vol56] Yu. A. Volkov. On deformations of a convex polyhedral angle. Uspehi Mat. Nauk (N.S.), 11(5(71)):209-210, 1956. MR0083765(18:758b)

Laboratoire Emile Picard, UMR CNRS 5580, UFR MiG, Université Paul Sabatier, 31062 Toulouse Cedex 9, France

E-mail address: schlenker@math.ups-tlse.fr 